ARTICLE

\title{
Multiple capsid protein binding sites mediate selective packaging of the alphavirus genomic RNA
}

\author{
Rebecca S. Brown (1) ${ }^{1}$, Dimitrios G. Anastasakis (10 ${ }^{2}$, Markus Hafner (1) ${ }^{2} \&$ Margaret Kielian (1) ${ }^{1 凶}$
}

The alphavirus capsid protein (Cp) selectively packages genomic RNA (gRNA) into the viral nucleocapsid to produce infectious virus. Using photoactivatable ribonucleoside crosslinking and an innovative biotinylated $\mathrm{Cp}$ retrieval method, here we comprehensively define binding sites for Semliki Forest virus (SFV) Cp on the gRNA. While data in infected cells demonstrate $\mathrm{Cp}$ binding to the proposed genome packaging signal (PS), mutagenesis experiments show that PS is not required for production of infectious SFV or Chikungunya virus. Instead, we identify multiple $\mathrm{Cp}$ binding sites that are enriched on gRNA-specific regions and promote infectious SFV production and gRNA packaging. Comparisons of binding sites in cytoplasmic vs. viral nucleocapsids demonstrate that budding causes discrete changes in $\mathrm{Cp}$-gRNA interactions. Notably, Cp's top binding site is maintained throughout virus assembly, and specifically binds and assembles with $\mathrm{Cp}$ into core-like particles in vitro. Together our data suggest a model for selective alphavirus genome recognition and assembly.

\footnotetext{
${ }^{1}$ Department of Cell Biology, Albert Einstein College of Medicine, Bronx, NY 10461, USA. ${ }^{2}$ Laboratory of Muscle Stem Cells and Gene Regulation, National

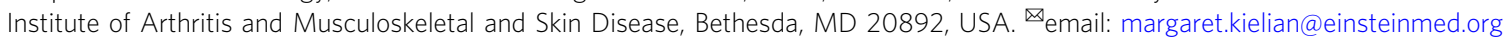


A lphaviruses are enveloped, highly organized RNA viruses that include a number of important human pathogens such as Chikungunya virus (CHIKV), Ross River virus (RRV), and Venezuelan Equine Encephalitis virus (VEEV) ${ }^{1}$. These viruses are transmitted by mosquito vectors and can infect a wide variety of mammalian and avian species. Alphaviruses are organized into complexes based on genetic and antigenic relationships ${ }^{2}$. While the general features of structure and lifecycle are shared, alphaviruses can differ in properties such as receptor usage, tissue tropism, and pathogenesis. Within the Semliki Forest virus (SFV) complex, CHIKV and RRV cause severe polyarthritis and have emerged to produce epidemics that affect millions of people globally ${ }^{3-5}$. To date, there are no specific antiviral treatments or licensed vaccines for any alphavirus.

Alphaviruses infect host cells by endocytic uptake and membrane fusion, thereby delivering the nucleocapsid (NC) core into the cytoplasm where replication takes place ${ }^{1}$. NC disassembly is thought to be mediated by interactions with ribosomes, resulting in the uncoating of the RNA genome ${ }^{6}$. The viral genome (gRNA) is an $\sim 11.5 \mathrm{~kb}$ single-stranded plus-sense RNA that is $5^{\prime}$-capped and contains a $3^{\prime}$-polyA tail. It is translated to produce the viral nonstructural proteins (nsP1-4), which assemble a membraneassociated replication complex (reviewed in refs. ${ }^{1,7}$ ). An antisense complement of the gRNA is synthesized and this negativesense RNA serves as the template for producing new gRNAs. In addition, an internal promoter drives the production of a smaller RNA that is identical in sequence to the last $\sim 1 / 3$ of the gRNA. This subgenomic RNA (sgRNA) is translated to produce the viral structural proteins: capsid protein $(\mathrm{Cp}), 6 \mathrm{~K} / \mathrm{TF}$, and the $\mathrm{E} 1, \mathrm{E} 2$, and E3 envelope proteins. Cp specifically interacts with the gRNA to assemble the NC (see below). Budding of progeny virus at the plasma membrane releases particles with icosahedral symmetry, containing 240 copies each of $\mathrm{Cp}, \mathrm{E} 1$, and E2, and $1 \mathrm{gRNA}^{1,8}$.

Alphaviruses have a robust growth cycle and produce particles with high specific infectivity, indicating strong quality control of particle assembly and genome packaging. However, it is not clear how the specific recognition and assembly of the viral RNA into $\mathrm{NC}$ occurs, given that $\mathrm{Cp}$ must select gRNA in the presence of a 3-4-fold molar excess of sgRNA produced from the replication complexes $^{9-12}$. In addition, while virus infection inhibits transcription of most cellular RNAs ${ }^{13,14}$, abundant host RNAs still remain in the infected cell, but are excluded from the viral particle $^{15}$. Serial passage under high multiplicity conditions can lead to the production of defective viral genomes that can be packaged into defective interfering (DI) particles. Such DI particles are missing portions of the gRNA, and are dependent on wild-type helper virus for their propagation. Mapping, sequence analysis, and mutagenesis studies of DI particles support the presence of a packaging signal (PS) in the gRNA that promotes its recruitment into virus particles (reviewed in Mendes and $\mathrm{Kuhn}^{16}{ }^{16}$. The nature and location of the PS appear to vary depending on the virus complex, with the VEEV complex and Sindbis virus (SINV) complex having a PS located in the nsP1 region ${ }^{17,18}$. In contrast, the PS for the SFV complex including CHIKV and RRV maps within the nsP2 region ${ }^{19,20}$. The evidence for a role of the PS in genome packaging is strongest for VEEV, where mutation of the PS in nsP1 leads to an $\sim 2-\log$ reduction in virus titer while still supporting growth to $\sim 10^{8} \mathrm{PFU} / \mathrm{ml}^{18}$.

While the inference is that the PS functions by interaction with the $\mathrm{Cp}$, as yet there is no evidence for Cp's direct binding to the PS in infected cells. Such studies are complicated by the fact that the highly basic nature of the $\mathrm{Cp}$ promotes its non-specific interaction with nucleic acids and proteins in vitro or in cell

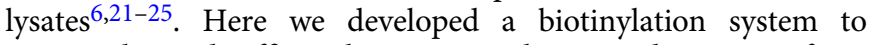
stringently and efficiently retrieve the capsid protein from SFV-infected cells or virus particles. We combined this with
PAR-CLIP (photoactivatable ribonucleoside crosslinking and immunoprecipitation $)^{26}$ to comprehensively map Cp interactions on the SFV genome with nucleotide precision. Our results identify $\mathrm{Cp}$ binding sites on the gRNA, rule out a role for the SFV and CHIKV PS, highlight changes in Cp-gRNA interactions during virus biogenesis, and identify a site on the gRNA that $\mathrm{Cp}$ preferentially binds and assembles with in vitro. Together our data support a model for alphavirus gRNA packaging and assembly that is mediated by $\mathrm{Cp}$ binding to multiple sites on the gRNA.

\section{Results}

AVI-tagged $\mathrm{Cp}$ is a tool to stringently and efficiently retrieve Cp. The alphavirus $\mathrm{Cp}$ is composed of an $\mathrm{N}$-terminal polybasic RNA-binding domain that mediates gRNA packaging, and a Cterminal protease domain that forms the outer NC shell (reviewed in Mendes and Kuhn ${ }^{16}$ ). The charged nature of the Cp ( $\mathrm{pI} \sim 10)$ promotes non-specific, electrostatically driven interactions, but wash conditions sufficient to disrupt them lead to poor protein retrieval by a variety of polyclonal and monoclonal $\mathrm{Cp}$ antibodies (RSB, unpublished observations). Moreover, prior studies showed that tagging $\mathrm{Cp}$ with proteins such as mCherry caused aberrant $\mathrm{Cp}$ localization and NC morphology (e.g. Zheng and Kielian ${ }^{27}$ ). In order to map Cp's binding sites on the gRNA, we devised a Cp retrieval strategy that takes advantage of the high affinity interaction of biotin with Streptavidin. The 13 residue minimal biotin acceptor peptide (mAVI tag; Fig. 1a) is specifically and efficiently biotinylated by the biotin ligase $\operatorname{Bir}^{28-31}$. We engineered the mAVI tag into the SFV infectious clone after $\mathrm{Cp}$ residue 95 (SFV-mAVI; Fig. 1a), a site previously shown to be permissive for small peptide insertions $s^{27,32}$. As a host cell for our studies we constructed a Vero cell line stably expressing humanized BirA in the cytoplasm (Vero BirA cells). Virus growth experiments showed that the mAVI tag was well tolerated, causing a modest decrease in maximal titers (Supplementary Fig. 1a). We observed no difference in virus or NC morphology by transmission electron microscopy (Supplementary Fig. 1b), or in the expression or localization of biotinylated Cp-mAVI vs. CpWT in infected cells (Fig. 1b and Supplementary Fig. 1c-e.) No change was detected in SFV-mAVI vs. WT specific infectivity as calculated by comparing the number of infectious particles to total particles (PFU:E2 ratio; Fig. 1c), thus indicating accurate packaging of the gRNA by Cp-mAVI. Using the biotin handle on $\mathrm{Cp}$, we optimized retrieval conditions with Streptavidin beads to efficiently and specifically pull-down $\mathrm{Cp}$. Under these stringent conditions (including $0.5 \%$ SDS and washing with $0.5 \mathrm{M} \mathrm{NaCl}$ ), $\mathrm{Cp}$ was retrieved from infected BirA cells only when biotin was present in the culture medium (Fig. 1d).

Cp binds many discrete sites across the genome. To define the Cp binding sites on the gRNA in infected cells at nucleotide resolution, we combined our optimized $\mathrm{Cp}$ retrieval system with the sensitive and specific PAR-CLIP method ${ }^{26}$. PAR-CLIP uses a photoactivatable ribonucleoside to crosslink labeled RNAs to their binding proteins, with up to 1000 -fold higher crosslinking efficiency compared to traditional CLIP-seq approaches. Crosslinking induces $\mathrm{T}$-to- $\mathrm{C}$ mutations during $\mathrm{cDNA}$ library generation, enabling precise identification of a protein's specific binding sites on the RNA ${ }^{26}$. Vero BirA cells were infected with SFV$\mathrm{mAVI}$ for $1.5 \mathrm{~h}$. The cells were then cultured in the presence of excess biotin to ensure robust Cp-mAVI biotinylation and the photoactivatable ribonucleoside 4-thiouridine (4SU) to label nascent RNAs. 4SU had no effect on virus growth. Because alphaviruses inhibit Pol II transcription early in infection ${ }^{13,14}$, this $4 \mathrm{SU}$ labeling strategy strongly biases labeling toward viral RNAs over cellular RNAs. At $7 \mathrm{~h}$ post-infection cells were 
a

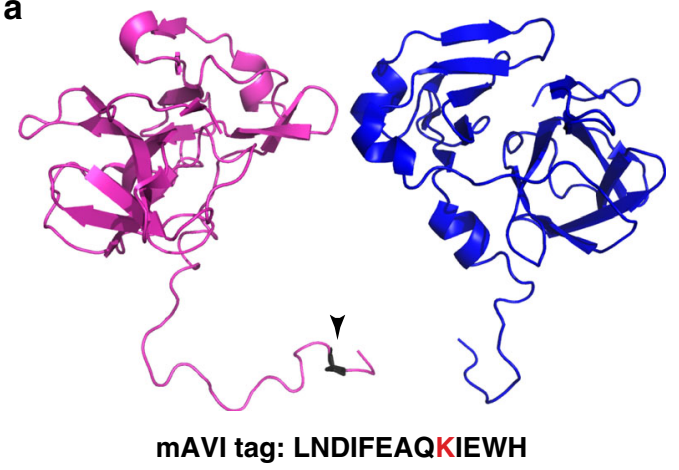

b

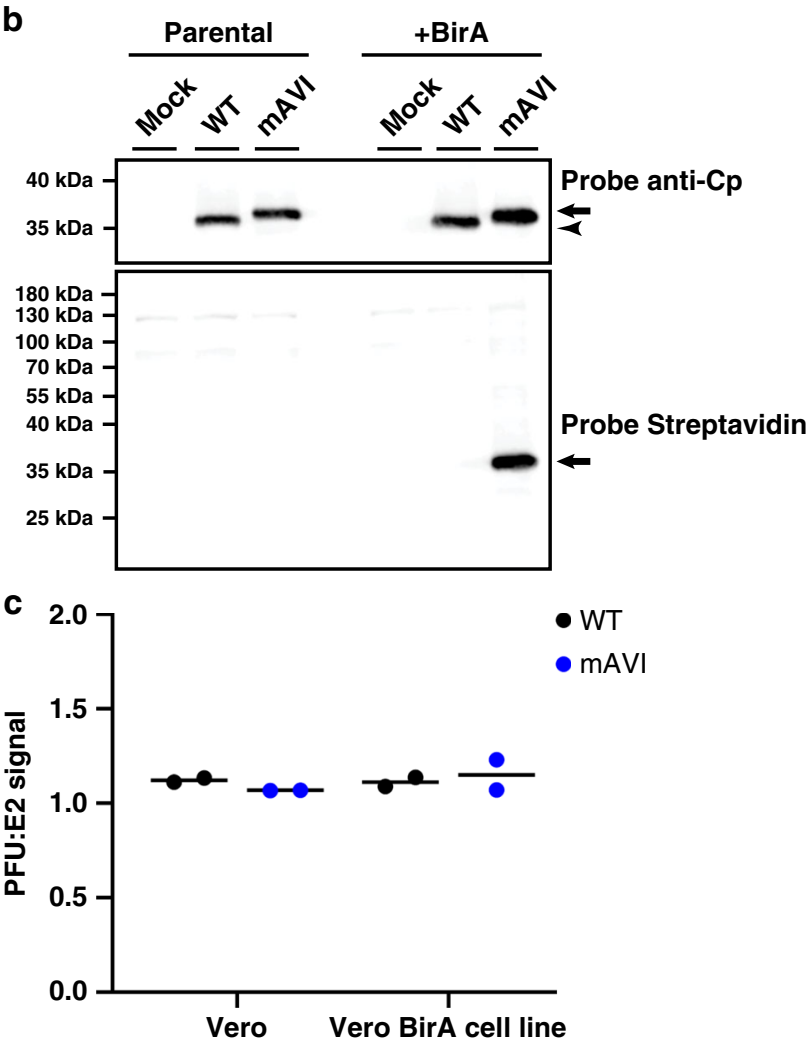

d
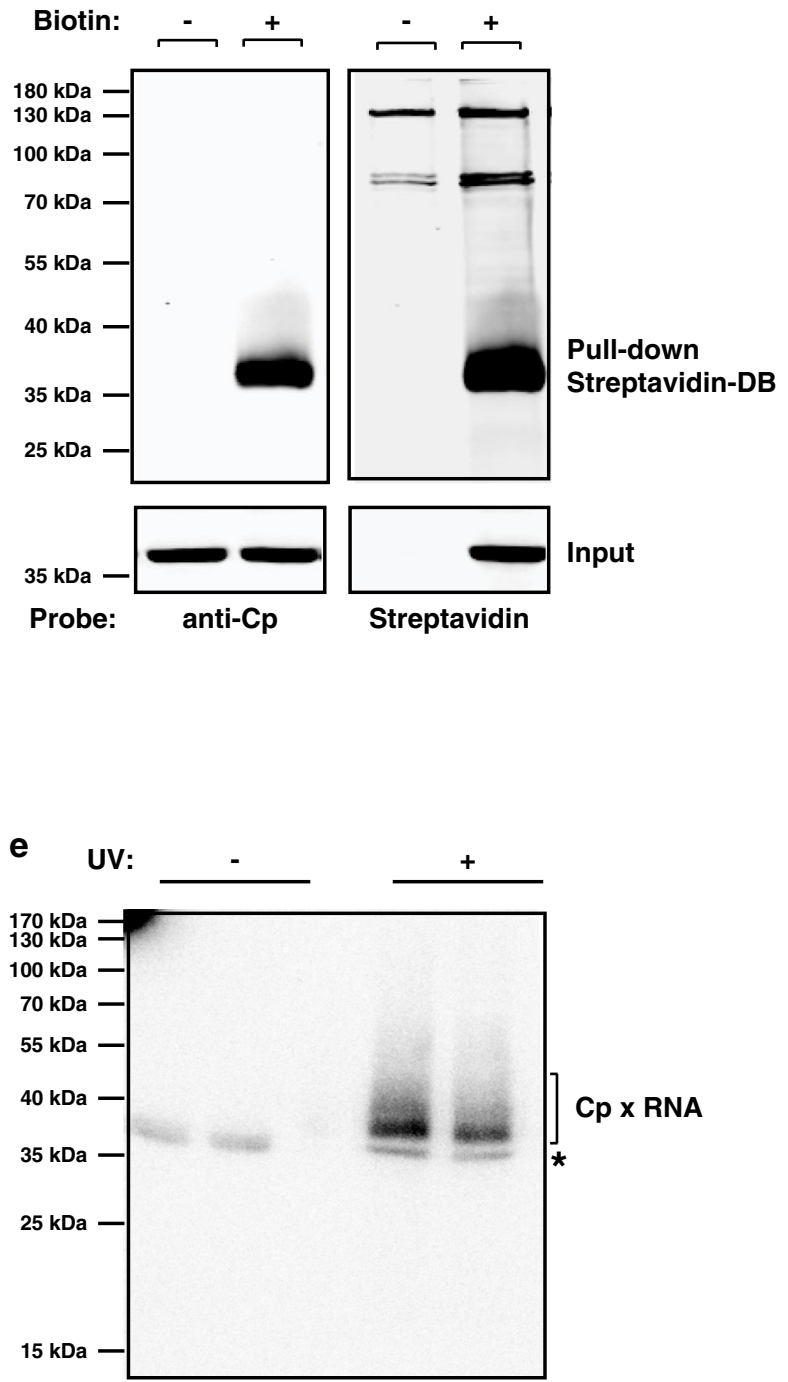

Fig. 1 Properties and retrieval of SFV Cp using mAVI tag. a Partial structure of Eastern Equine encephalitis virus $C p$ dimer encompassing (on the left) $C p$ residues 85-261 (PDB: 6MX7) ${ }^{74}$. Arrowhead indicates the homologous location where the mAVI tag was inserted in SFV Cp. The highlighted lysine residue within the $\mathrm{mAVI}$ tag sequence is the target for BirA biotinylation. b Vero parental or Vero BirA (+BirA) cells were mock infected, infected with SFV WT, or infected with SFV mAVI. At $7.5 \mathrm{hpi}$, lysates were harvested, subjected to SDS-PAGE, and analyzed by western blot using a polyclonal antibody against Cp or a Streptavidin Alexa-680 probe. Arrowhead indicates Cp WT, and arrows indicate Cp-mAVI. The images are representative examples from $n=2$ biological replicates. c Specific infectivity was measured as the ratio of the number of infectious particles (determined by plaque assay) to the number of total particles (determined by quantitative western blot of purified virus using an E2-specific antibody, see Methods section). Individual data points from two biological replicates are shown and the black line represents their mean. Units represent ratio of PFU/E2 signal. d Vero BirA cells were grown in biotindepleted media for 3 days (-biotin) or grown in normal media (+biotin). Cells were infected with SFV mAVI, and lysates were harvested at 7.5 hpi, retrieved with Streptavidin DynaBeads (SA-DB), and analyzed by SDS-PAGE and western blot using a monoclonal antibody against Cp or a Streptavidin Alexa-680 probe. The images are representative examples of $n=2$ biological replicates. e Autoradiogram of $C p$-RNA crosslinked adducts retrieved with SA-DB from Vero BirA cells infected with SFV mAVI (representative of $n=2$ biological replicates). Asterisk indicates an infection- and UV-independent band (Supplementary Fig. 1e). See also Supplementary Fig. 1.

irradiated with UV ( $\lambda 368 \mathrm{~nm})$ to crosslink RNAs with bound proteins, lysed, and RNAs digested with RNaseT1 to produce footprints protected by RNA-binding proteins. The total cellular pool of Cp-mAVI-biotin was then retrieved with Streptavidin beads, and crosslinked RNAs were $5^{\prime}$-end labeled with $\gamma^{-32} \mathrm{P}$-ATP and subjected to SDS-PAGE followed by transfer to a nitrocellulose membrane. The resulting Cp-RNA adducts were only detected upon UV irradiation and were the only UV-dependent crosslinked products that were retrieved (Fig. 1e and Supplementary Fig. 1f).

The RNAs crosslinked to $\mathrm{Cp}$ were purified and converted into cDNA libraries and sequenced using the Illumina MiSeq Platform (see Methods section for details). From two biological replicates we obtained 1,384,633 and 3,213,621 sequence reads of which 121,119 and 284,837 mapped to the viral genome, respectively. For further analysis we only considered the 105,920 and 
a

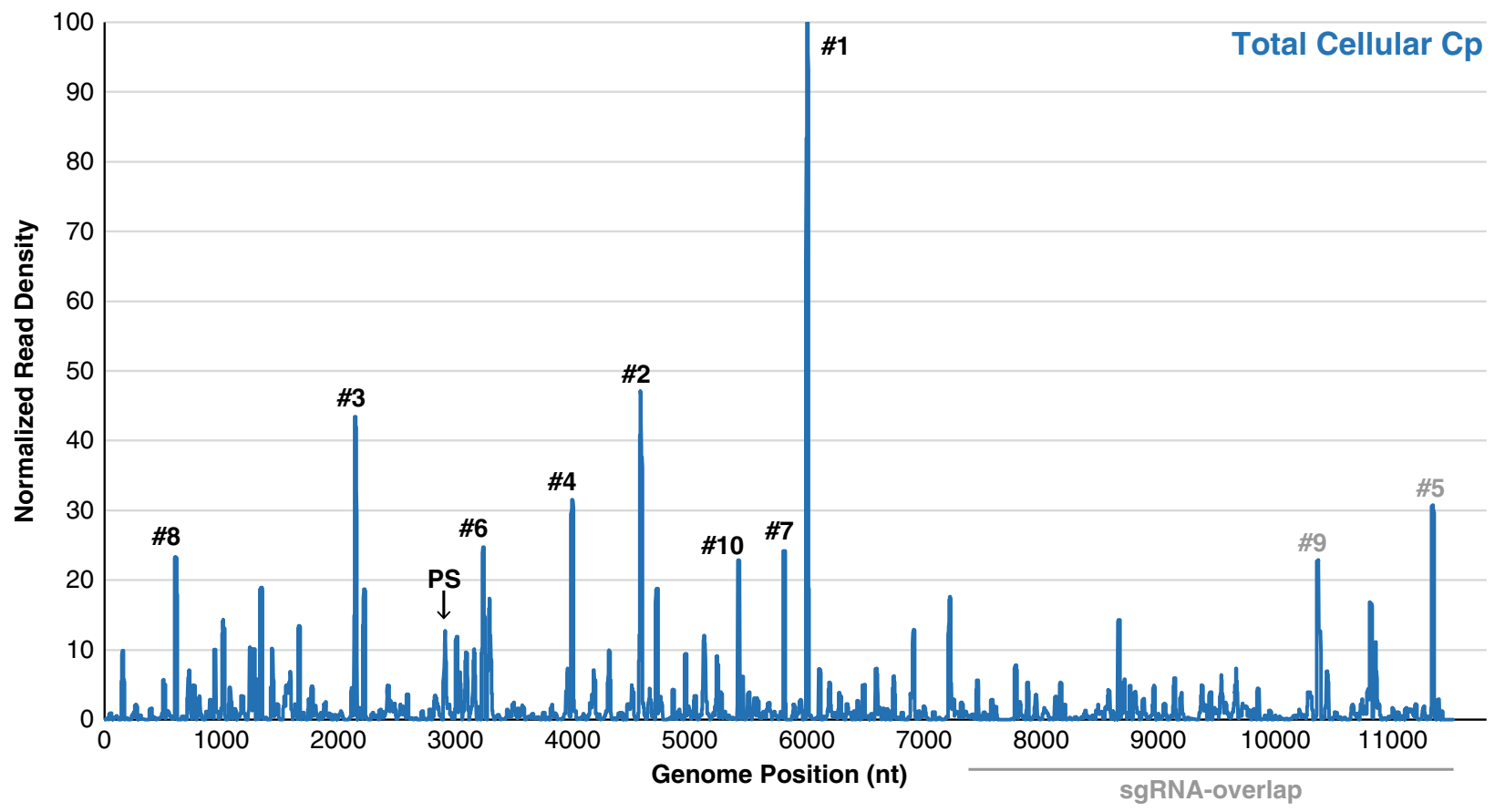

b
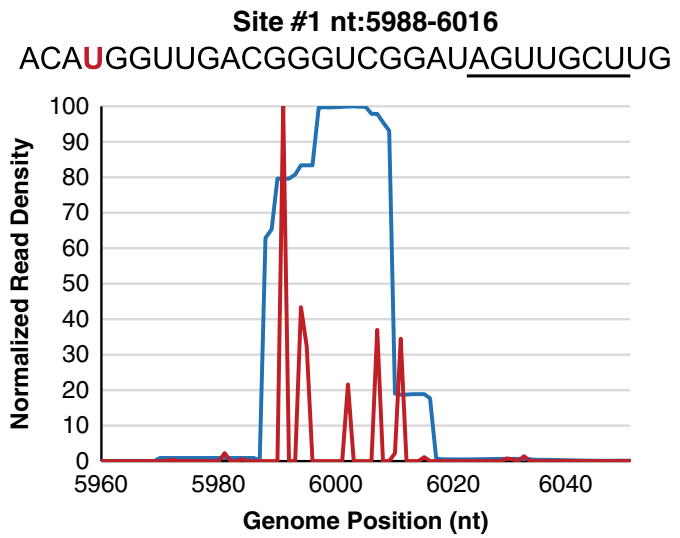

C AAAAUCCCUUGUAUGCCCCUGCGUCGGAGCACGUG

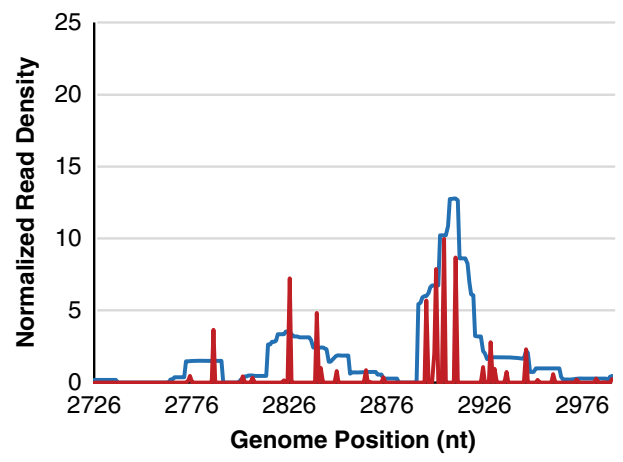

233,188 sequence reads, respectively, that contained the diagnostic T-to-C mutation introduced during cDNA library construction of 4SU-labeled and crosslinked RNA. This allowed us to (a) remove background sequences from co-purifying, noncrosslinked fragments from abundant RNAs and (b) identify the crosslinking site at nucleotide resolution. Comparison of the crosslinked sequence reads revealed an excellent correlation for
Site \#2 nt:4563-4592

ACAUGAGGACGGCUGUGGAGUUGCUCAAUG

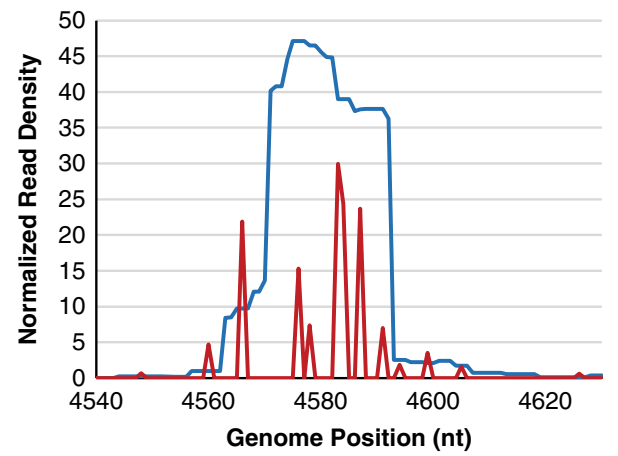

d

Site \#1 ACAUGgUUGACGGGUCGGAUAGUUGCUUG

Site \#2 ACAUGAgGACGGCUGUGGAGUUGCUCAAUG

e

Site \#1

SFV

GETV

ONNV

MAYV

CHIKV

RRV 
Fig. 2 Cp's genomic RNA-binding sites in cells. a Normalized read density from two independent PAR-CLIP experiments plotted across genome nucleotide position. Reads were normalized to the nucleotide position with the highest read frequency within the library, which were read values of 14,134 and 13,343, before averaging. Arrow indicates Cp's specific binding site within the Full PS, \#1 indicates site \#1, \#2 indicates site \#2, etc. Gray line represents the sgRNA-overlapping region of the gRNA. b Normalized read density (blue lines) across site \#1 (nt:5988-6016; left) and site \#2 (nt4563-4592; right), and the normalized read frequencies of the $T$-to- $C$ mutations (red lines). $T$-to- $C$ frequencies were normalized to the $T$ with the highest $C$ mutation frequency within each library replicate, which were frequencies of 5386 and 4750, before averaging. The T/U residue with the most $C$ mutations for each site is highlighted in red within their respective sequence. The seven identical nucleotides between site \#1 and 2 are underlined. $\mathbf{c}$ As in $\mathbf{b}$, but for the Full PS (nt:2726-2991). The sequence corresponding to the Cp-PS binding site (nt:2892-2926) is shown at the top, with the T/U residue with the most C mutations highlighted in red. d Site \#1 and \#2 sequences. Bolded nucleotides are either "UUG" or "UGG" motifs. The seven identical nucleotides between site \#1 and 2 are underlined. e Sequence alignment of site \#1 with the homologous sites in SFV complex members GETV (AY702913.1), CHIKV (EF452493.1), MAYV (AF237947.1), ONNV (M20303.1), and RRV (GQ433359.1). Asterisks indicate nucleotide identity, and periods indicate nucleotide similarity. See also Supplementary Figs. 2-4, and Supplementary Data 1 and 2.

Approximately $\sim 100$ additional Cp interaction sites could be observed on the gRNA, but with significantly lower read densities (Supplementary Data 2).

Analysis of the nucleotide composition of Cp's top 58 binding sites revealed that these sequences were relatively U-rich and Apoor (Supplementary Fig. 2a). In contrast, sequences negative for $\mathrm{Cp}$ binding in either replicate were relatively A-rich. Previous studies show that PAR-CLIP can identify binding sites of all compositions. For example, binding sites for the HIV-Gag protein are not U-rich ${ }^{33}$, while CNBP and DHX36 selectively bind G-rich sites $^{34,35}$. Thus our results suggest that the SFV Cp does in fact preferentially bind $\mathrm{U}$-rich sequences in the cell. A computational search $^{36}$ for sequence motifs enriched in the top $58 \mathrm{Cp}$ binding sites revealed that GC- and UG-based motifs were common, but did not identify a specific sequence motif from these 58 sites (Supplementary Fig. 3a).

The published DI-RNA studies mapped the SFV PS to a 266-nt region located within the nsP2 gene (nt:2726-2991; here termed Full PS), suggesting a role for this region in gRNA packaging ${ }^{19,20}$. Our PAR-CLIP data showed that $\mathrm{Cp}$ bound a 35-nt region toward the $3^{\prime}$ end of this region (nt: 2892-2926; here termed CpPS binding site), with weaker Cp binding also observed within nt: 2815-2856 (Fig. 2c). This is the first direct demonstration of in cell interaction of $\mathrm{Cp}$ within the Full PS region. However, the CpPS binding site was not the top binding site on the gRNA, with 20 other sites showing higher binding (Fig. 2a, b).

We re-analyzed the Cp binding sites on the gRNA using the CpPS binding site as a lower cutoff. Within these 21 high affinity sites there was a stronger trend toward $\mathrm{Cp}$ binding U-rich and A-poor sequences (Supplementary Fig. 2a). Sequence motif analysis of the top 21 sites showed an enrichment for UUG and UGG-trinucleotide motifs (Supplementary Fig. 3a). Secondary structure predictions for the top 21 sites predicted all 21 RNAs would adopt stem-loop structures, but of varying sizes (Supplementary Fig. 3b) ${ }^{37}$.

Manual inspection of Cp's highest affinity sites revealed that Cp's top two binding sites (nt: 5988-6016 and nt: 4563-4592; Fig. 2 b, sites \# 1 and \#2) are very similar in sequence and contain UUG and UGG motifs (Fig. 2d, bolded). Furthermore, sites \#1 and \#2 share an identical stretch of seven-nucleotides not present at any other location within the gRNA (Fig. 2b, d, underlined). Sequence alignment analysis showed that site \#1's sequence is hyperconserved across several other viruses in the SFV complex compared to their overall sequence identity (Fig. 2e), while site \#2 and 12 other top binding sites are hyperconserved in CHIKV as well (Supplementary Fig. 4a). Closer inspection of site \#1 and \#2's predicted secondary structures revealed similar stem-loop structures containing tandem G:U wobble base pairs within the stem (Supplementary Fig. 3b). Tandem G:U wobble base pairs were also predicted to form in the stems of sites \#8, \#14, and \#15 (Supplementary Fig. 3b). While these results are interesting, we note that such predictions are limited because they do not account for the sequence's placement within the context of the gRNA $(\sim 11.5 \mathrm{~kb})$, or for the effects of Cp binding or the complex cellular environment. In keeping with this, secondary structure predictions using longer fragments centered around each of the 17 input sequences produced variations in the predicted stemloop structures (as shown in Supplementary Fig. 3c, d for Sites\#1 and \#2). Thus direct experimental evidence will be needed to determine the structures adopted by these $\mathrm{Cp}$ binding sites in the gRNA within the infected cell and virus particle.

Cp preferentially binds sequences unique to the genomic RNA. The sgRNA is identical in sequence to the last $\sim 1 / 3^{\text {rd }}$ of the gRNA and is present in 3-4X molar excess over it in infected cells ${ }^{9-11}$. It has therefore been hypothesized that $\mathrm{Cp}$ binding sites specific to the gRNA, such as the PS, dictate selective packaging in infected cells. Inspection of our PAR-CLIP data suggested that Cp binding was biased toward the first $\sim 2 / 3^{\text {rd }}$ of the genome (Fig. 2a). To formally test this, we summed the number of observed reads at each nucleotide position and categorized them as mapping to the gRNA-specific region vs. mapping to the sgRNA-overlapping region (Fig. 3a). We performed a $\chi^{2}$ analysis to test whether the observed summed read distributions were statistically different from our null hypothesis, in which random $\mathrm{Cp}$ binding would result in $\sim 2 / 3$ of the summed reads mapping within the gRNAspecific region and $\sim 1 / 3$ within the sgRNA region. The results showed that for each replicate, observed read distributions mapping to gRNA-specific regions were significantly higher than expected $(p<0.0001$; Fig. 3a). This result was not due to a nucleotide bias as the gRNA-specific and sgRNA sequences have nearly identical compositions (Fig. 3b). The enriched binding of $\mathrm{Cp}$ to gRNA-unique sequences suggests a direct molecular rationale for selective $\mathrm{Cp}$ recognition and packaging of gRNA vs. sgRNA.

Cp binding sites promote infectious virus production. We set out to assess the significance of Cp's top binding sites on genome packaging by mutating them and measuring the effect on infectious virus production. All mutations were designed to strictly maintain amino acid sequence identity, disrupt predicted secondary structures, and minimize rare codon usage and changes in dinucleotide frequencies (Supplementary Data 3). We first mutated the Full PS previously identified by DI-RNA studies (see Supplementary Fig. 3e for predicted effects on PS secondary structure). To our knowledge, while such mutational analyses have been performed for the SINV and VEEV PS ${ }^{18}$, this is the first direct test of PS function for any SFV complex virus. We infected Vero cells with the SFV WT or Full PS mutant and measured infectious virus production over time. The Full PS mutant did not affect virus growth compared to WT (Fig. 4a). 
a
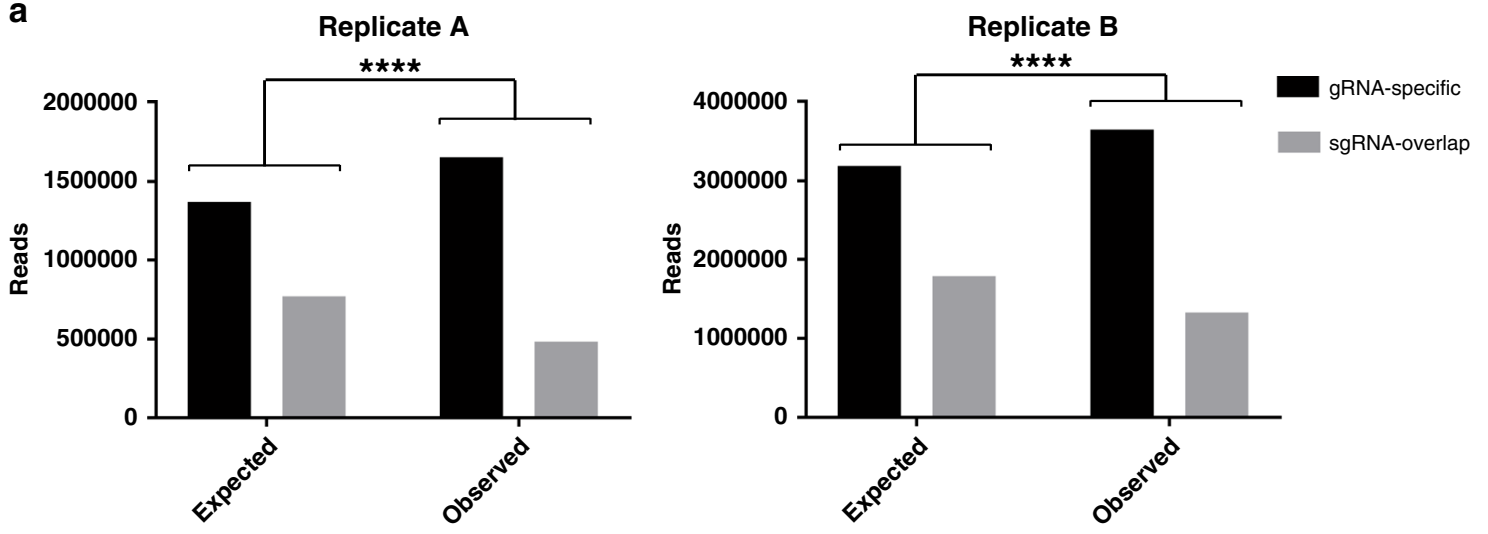

b

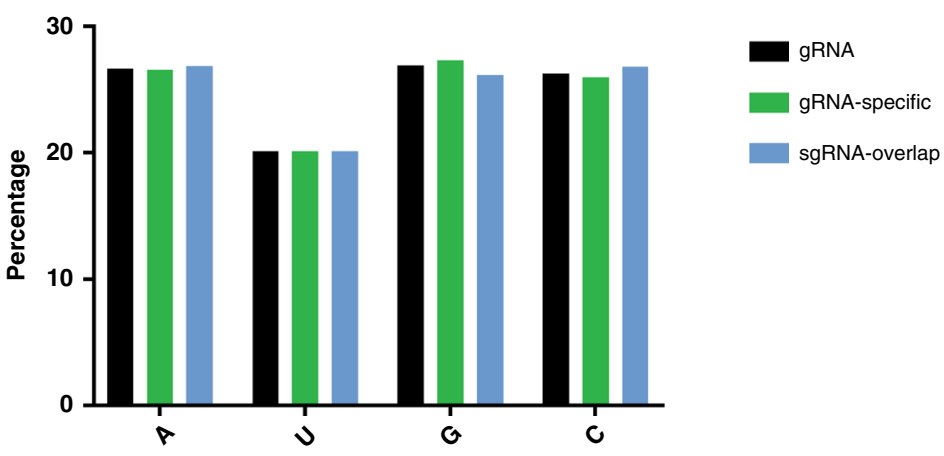

Fig. 3 Cp preferentially binds genome-specific sequences. a $\chi^{2}$ analysis test for biological replicate libraries (a, b) comparing the expected vs. observed number of sequence reads summed per nucleotide position mapping to gRNA-specific sequences (black) or sequences that overlap with the sgRNA (gray). Two-sided, $\mathrm{df}=1,{ }^{\star \star *} p<0.0001$. b Nucleotide composition of the gRNA, gRNA-specific sequences, and sequences that overlap with the sgRNA. See also Supplementary Data 2.

This is consistent with our PAR-CLIP data, which demonstrated that $\mathrm{Cp}$ does not extensively interact with the PS region, and more efficiently binds $\sim 20$ other sites on the gRNA.

To test if other alphaviruses in the SFV complex were dependent on the previously identified PS, we mutated the region of the CHIKV gRNA corresponding to the SFV DI-RNA-derived Full PS (Supplementary Fig. 4b and Supplementary Data 3). Similar to our results with SFV, we observed no effect of mutation of the CHIKV Full PS on infectious CHIKV production (Fig. 4b).

We next focused on a series of mutational analyses of the top gRNA-specific binding sites. Mutation of Cp binding sites \#1 and \#2 (Double mutant) had a minor effect on infectious virus production ( $\sim 4$-fold reduction at $5 \mathrm{hpi}$; Fig. $4 \mathrm{c})$. Mutagenesis of sites \#1-4 plus the Cp-PS binding site (Quintuple mutant) caused a more pronounced effect on infectious virus production $(\sim 8$-fold reduction at $5 \mathrm{hpi}$; Fig. $4 \mathrm{c}$ ); growth at later time points was comparable to that of WT. We then tested if packaging could occur through cooperation among multiple $\mathrm{Cp}$ binding sites on the gRNA. Such a multi-site packaging mechanism has been described for some plus-sense RNA viruses ${ }^{16,38}$. Using the $35-n t$ Cp-PS binding site as a threshold, we mutated 17 gRNA-specific binding sites including Cp-PS, representing Cp's highest affinity binding sites on the gRNA (Supplementary Data 3). Other than the site within the PS, none of these have been previously implicated in any stage of the alphavirus life cycle. The 17-site mutant produced significantly less infectious virus compared to WT at early time points of virus production (Fig. 4c; $~ 74$-fold reduction at $5 \mathrm{hpi}$, and $\sim 6$-fold reduction at $6 \mathrm{hpi}$ ). Combining the 17-site mutant with the full PS mutant produced no additional decrease in virus production. The 17-site mutant phenotype became more moderate at late times of virus production (Fig. 4c; 3-fold reduction at 8 and 10 hpi), suggesting that these sites are most important at early time points in virus assembly when $\mathrm{Cp}$ levels are limiting. This is consistent with a multi-site packaging mechanism where high affinity binding sites on the gRNA have a greater impact on gRNA recognition and NC assembly at early infection times when Cp levels are lower ${ }^{39}$.

To determine if the 17-site mutant specifically impacted gRNA packaging and virus assembly vs. aspects of virus replication, we first checked viral protein expression levels by western blot. We observed comparable levels of both nsP2 and Cp between WT vs. 17-site mutant-infected cells (Fig. 4d). We next used RT-qPCR to measure cellular gRNA and sgRNA levels over the course of virus infection, using primers that either specifically amplify the gRNA or amplify both gRNA and sgRNA [gRNA+sgRNA] due to their overlapping sequences. No significant difference was observed in cellular gRNA or [gRNA+sgRNA] levels between WT and 17-site mutant-infected cells from 3 to 7 hpi (Fig. 4e). Thus, although the gRNA mutations significantly reduce infectious virus production, this was not due to defects in viral RNA replication or protein expression.

We next asked whether the reduction in infectious particles could be due to a decrease in genome packaging in the 17-site mutant. We infected cells, purified SFV WT or 17-site mutant viruses, and quantitated viral gRNA and [gRNA+sgRNA] levels in the released particles. There was a significant decrease in gRNA in the 17-site mutant virus collected at 5 hpi ( $>10$-fold reduction; Fig. 4f). There was no significant difference in gRNA levels at 7 hpi, or in [gRNA+sgRNA] levels for either time point (Fig. 4f). The $\sim 10$-fold reduction in gRNA levels in the 17-site mutant virus at 5 hpi correlated well with the $\sim 10$-fold reduction in infectious particles observed in a parallel sample at $5 \mathrm{hpi} \mathrm{(Fig.} 4 \mathrm{~g}$ ).

At these time points and multiplicities we were unable to directly quantitate particle numbers by quantitating $\mathrm{E} 2$ in released viral particles. Since it was previously demonstrated that 

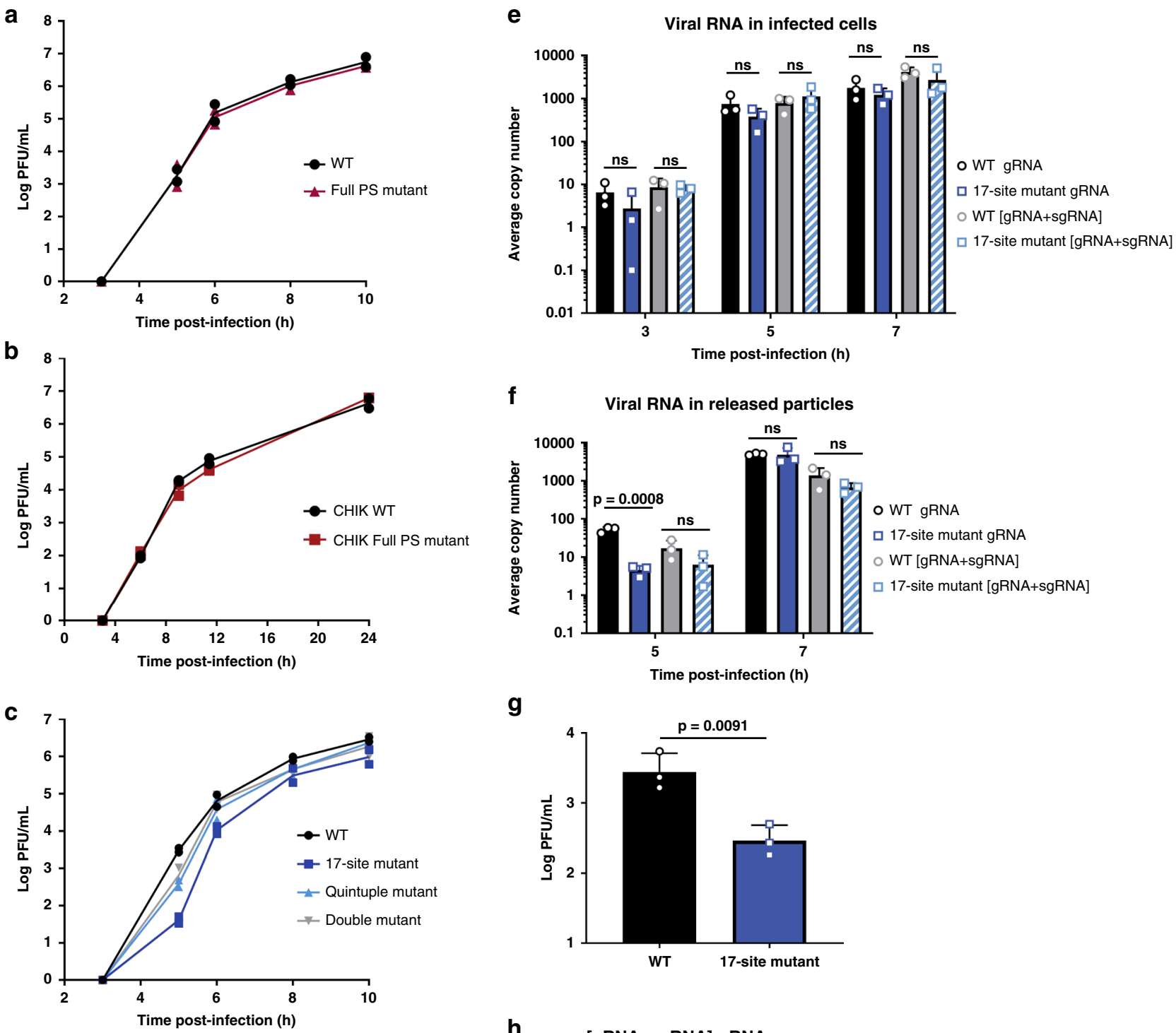

g

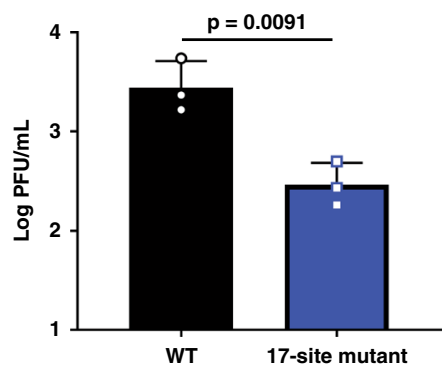

h
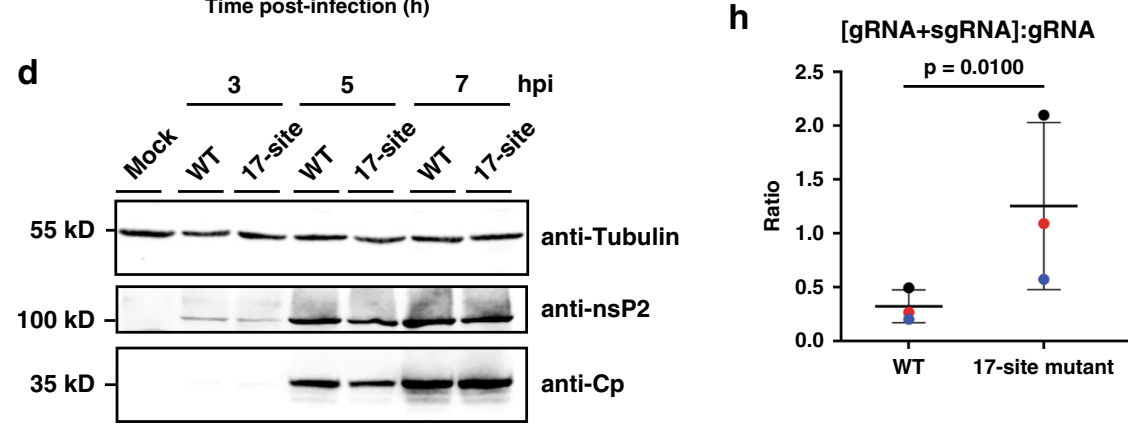

a decrease in alphavirus gRNA packaging can result in increased packaging of the sgRNA ${ }^{11,18}$, we analyzed if there was more sgRNA packaged into 17-site mutant particles. Results showed that even though the gRNA levels were significantly reduced in the mutant virus particles at $5 \mathrm{hpi}$, their [gRNA+sgRNA] levels were not significantly different from those of WT virus (Fig. 4f). Comparing the ratio of [gRNA+sgRNA] to gRNA between the WT and 17-site mutant particles showed a significantly higher ratio ( 4-fold) for the 17-site mutant compared to WT (Fig. $4 \mathrm{~h}$ ), suggesting a higher proportion of sgRNA in the mutant particles. These data thus suggest that the decrease in gRNA and infectious particles observed for the 17-site mutant is at least in part caused by increased non-specific packaging of the sgRNA.
Together, our results rule out a significant role of the previously proposed DI-RNA-derived PS in infectious virus production for the SFV complex. Instead, they support a mechanism in which alphaviruses package their gRNAs through $\mathrm{Cp}$ interaction with multiple high affinity sites present in the gRNA-specific region of the genome.

Changes in Cp:gRNA interactions during virus exit. Our PARCLIP results were obtained by analysis of the total cellular Cp in infected cells, a pool that includes both unassembled $\mathrm{Cp}$ and assembled NC (Fig. 5a). To examine different stages of virus assembly, we compared these total cellular Cp results with 
Fig. 4 Mutation of multiple $\mathbf{C p}$ binding sites decreases infectious virus production. a Vero cells were infected with SFV WT or Full PS mutant at MOI = 0.01. Media from the indicated time points were titered by plaque assay. Individual points from $n=2$ biological replicates are plotted and the line represents their mean. $\mathbf{b}$ As in a, but with CHIKV WT and CHIKV Full PS mutant at MOI $=0.01$. c As in a and $\mathbf{b}$, but with SFV WT, 17-site mutant, Quintuple mutant (sites \#1-4 and Cp-PS binding site), and Double mutant (sites \#1 + 2). d Lysates from Vero cells mock infected, or infected with SFV WT or the 17-site mutant at $\mathrm{MOI}=10$ were analyzed by western blot for $\beta$-tubulin, nsP2 or $\mathrm{Cp}$. Representative images of $n=2$ biological replicates. e Total cellular RNA was extracted from Vero cells infected with SFV WT or 17-site mutant at $\mathrm{MOI}=0.01$. gRNA and [gRNA+sgRNA] copy numbers in infected cells were determined by RT-qPCR and represent the average per $5 \mathrm{ng}$ of total RNA. Individual data points from $n=3$ biological replicates are plotted. Error bars indicate the mean \pm s.d. "ns" (not significant) from two-tailed t-tests. f Media from Vero cells infected with SFV WT or 17-site mutant at MOI $=0.01$ were pelleted to isolate virus particles. gRNA and [gRNA+sgRNA] copy numbers were determined as in e and represent the average copy number per $10 \%$ of the pelleted samples. Individual data points from $n=3$ biological replicates are plotted. Error bars indicate the mean \pm s.d. $P$-value and "ns" from twotailed $t$-tests. $\mathbf{g}$ SFV WT and 17-site mutant samples from the 5 -h time point in $\mathbf{f}$ were titered by plaque assay. Individual data points from $n=3$ biological replicates are plotted. Erorr bars represent the mean \pm s.d. P-value from two-tailed ratio paired $t$-test. $\mathbf{h}$ The ratio of [sgRNA+gRNA] to $g R N A$ levels from the $5 \mathrm{~h}$ time point in $\mathbf{f}$ for SFV WT and 17-site mutant. The thick black horizontal bar represents the mean and the thin horizontal bars represent the s.d. Paired replicate ratios from the $n=3$ biological replicates in $\mathbf{f}$ are plotted in the same color. P-value from two-tailed ratio paired $t$-test. See also Supplementary Fig. 4 and Supplementary Data 3.

PAR-CLIP analyses performed on cellular and viral NC produced in 4SU-labeled cells (Fig. 5a). For cellular NC, UV-crosslinking was performed on infected cells as above, followed by cell lysis and subsequent $\mathrm{NC}$ purification by sucrose gradient sedimentation (Supplementary Fig. 2b). For viral NC, released virus particles were pelleted, UV-crosslinked, and then lysed. Biotinylated Cp-RNA adducts from viral and cellular NCs were retrieved with Streptavidin beads (Supplementary Fig. 2c), as described above. As observed for the total cellular $\mathrm{Cp}$, retrieval was strictly dependent on UV irradiation (Fig. 5b). PAR-CLIP libraries were generated from two biological replicates for each sample type, and reads were processed as above. Biological replicates correlated strongly (Pearson correlation coefficients of $r=0.8655$ for cellular $\mathrm{NC}$ and $r=0.9459$ for viral NC) and thus we averaged the duplicate libraries and plotted normalized read density across the gRNA sequence (Fig. 5c, d).

Overall, Cp:gRNA interactions appear similar across virus assembly states, with many of the same binding sites maintained over the course of assembly (Fig. 5c, d, arrowheads). Cp's highest affinity site in the total cellular Cp sample (Fig. 2b, \#1) is also the highest affinity site in cellular NCs (Fig. 5c, termed NC\#1) and viral NCs (Fig. 5d, termed V\#1), demonstrating that $\mathrm{Cp}$ preferentially binds this specific RNA sequence across virus assembly states. Additionally, the third best bound site in the total cellular Cp population (Fig. 2 b, \#3) was also the third best bound site in cellular NC (Fig. 5c, NC\#3) and the second-best bound site in viral NC (Fig. 5b, V\#2). Cp's top four binding sites in the total cellular Cp population also showed strong binding within cellular $\mathrm{NC}$ and viral NC (Figs. $2 \mathrm{~b}$ and $5 c$, $d$ and Supplementary Data 2), further emphasizing Cp's affinity for these RNA sequences. In addition, all of the assembly states displayed enriched binding of Cp to gRNA-specific sequences (Fig. 5d).

Distinct changes were observed between cellular NC and viral NC (Fig. 5c, black arrows). For example, Cp's second highest affinity site in cellular NC is located at nt: 2997-3016 (Fig. 5c, $\mathrm{NC} \# 2$ ). Upon virion budding, this binding site drops below the top 50 binding sites within the viral NC. This pattern of strong Cp:gRNA binding events in cellular NC that become less prominent in viral NC is also observed for cellular NC sites NC\#4 (nt:6096-6115), NC\#5 (nt:4954-4972), and NC\#8 (nt:5569-5706), among others (Fig. 5c, arrows). In contrast, certain sites such as site \#1 are bound in all of the assembly states we assayed, suggesting these sites may play critical roles in NC assembly and structure.

Specific binding of $\mathrm{Cp}$ and gRNA site \#1 in vitro. Cp's high promiscuity for nucleic acids and anionic molecules in vitro induces its assembly into spherical core-like particles (CLPs) that strongly resemble viral NCs in overall structure and symmetry ${ }^{21,23,24}$. CLP assembly is largely driven by electrostatic interactions with little discrimination among anionic cargoes ${ }^{25,40}$. Because of this, it is unclear whether specific RNA sequences can drive CLP and NC assembly. To date, no discrete RNA sequence has been shown to be specifically recognized by full-length $\mathrm{Cp}$ both in infected cells and in vitro. Our PAR-CLIP data showed that Cp's top binding site on the gRNA across all stages of virus assembly was the same sequence (\#1 in Figs. $2 a$ and $5 c$, d). This suggested that site \#1 may encompass a distinct RNA motif recognized by $\mathrm{Cp}$. To assess this, we tested whether $\mathrm{Cp}$ specifically binds and/or assembles with site \#1 in vitro. We recombinantly expressed and purified N-terminally Strep-tagged SFV Cp (Supplementary Fig. 5a). To specifically test binding, Cp was immobilized on Streptactin beads to prevent CLP assembly. Immobilized $\mathrm{Cp}$ was then incubated with ${ }^{32} \mathrm{P}$-labeled RNAs representing site \#1 or a mutated version identical to that generated in the virus mutant, either alone (first lanes) or in the presence of increasing amounts of an unlabeled random RNA pool (Fig. 6a; upper panels). Both RNAs bound to Cp, but the mutant site \#1 RNA was more efficiently competed by random RNA. However, when the binding experiment was performed using $\mathrm{Cp}$ preincubated with poly(I:C) to shield non-specific electrostatic interactions, Cp specifically bound site \#1 RNA even in the presence of increasing concentrations of random RNA (Fig. 6b; lower panel, left side). In contrast, under the same conditions $\mathrm{Cp}$ inefficiently bound the mutant RNA, and its binding was entirely competed by random RNA (Fig. 6b; lower panel, right side). Thus, Cp specifically and directly binds gRNA site \#1 both in cells and in vitro, demonstrating that site \#1 encompasses a motif that $\mathrm{Cp}$ specifically recognizes.

We next assessed whether Cp could specifically assemble with site \#1 into CLPs. We found that poly(I:C) alone induced CLP formation, in keeping with the ability of long anionic polymers such as heparin to promote CLP formation ${ }^{25}$. Therefore, to shield non-specific electrostatic interactions we instead used a 10 nucleotide DNA oligo (here termed 10mer) that is below the length needed to induce CLP formation ${ }^{21}$. We confirmed that CLPs did not form with the $10 \mathrm{mer}$ by negative stain electron microscopy (EM), which showed amorphous material but no detectable CLPs (Fig. 6b; upper panels). The addition of site \#1 RNA produced abundant spherical CLPs of $\sim 40 \mathrm{~nm}$ in diameter (Fig. 6b; lower), consistent in appearance to that of previously published CLPs ${ }^{21,23}$. We then quantitated CLP formation by sucrose gradient sedimentation and detection of Cp by ELISA (Fig. 6c and Supplementary Fig. 5b). As predicted, Cp alone or incubated with 10mer (1:20 Cp:10mer) showed most Cp remained unassembled at the top of the gradient (Fig. 6c). Cp incubated with site \#1 RNA alone 
a

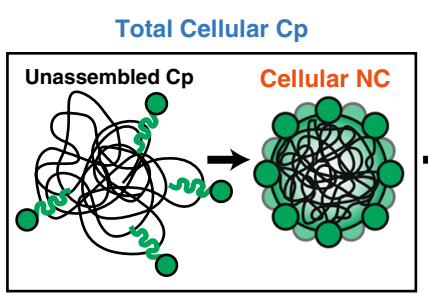

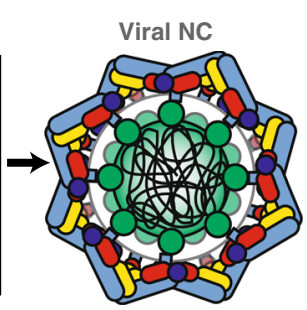

b

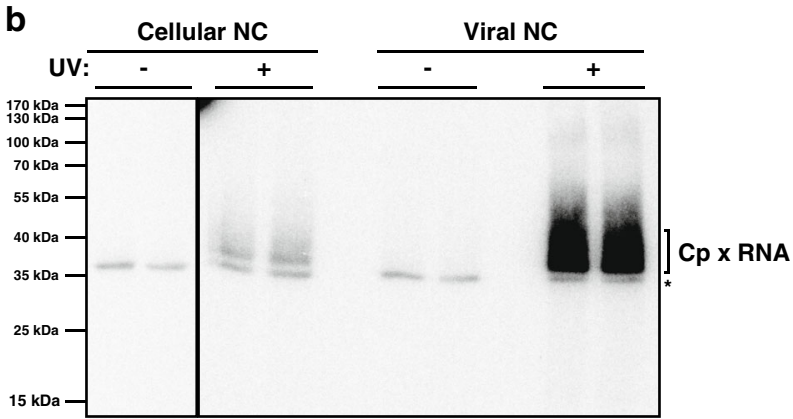

C

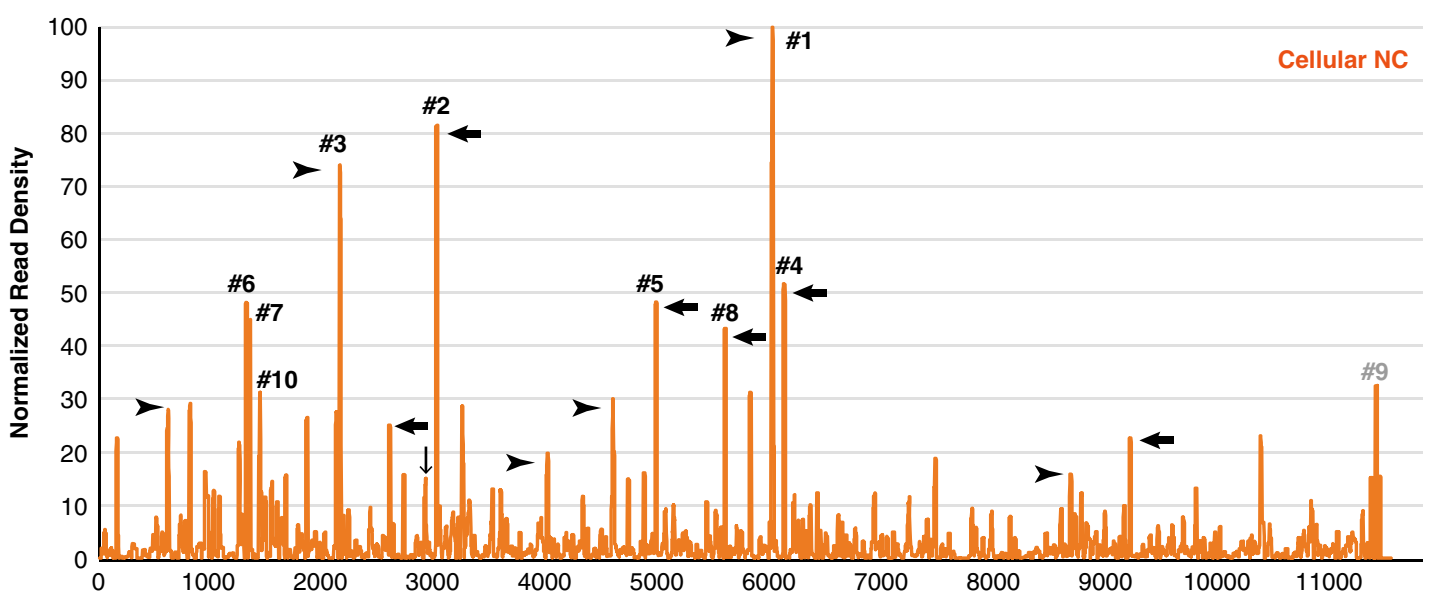

d

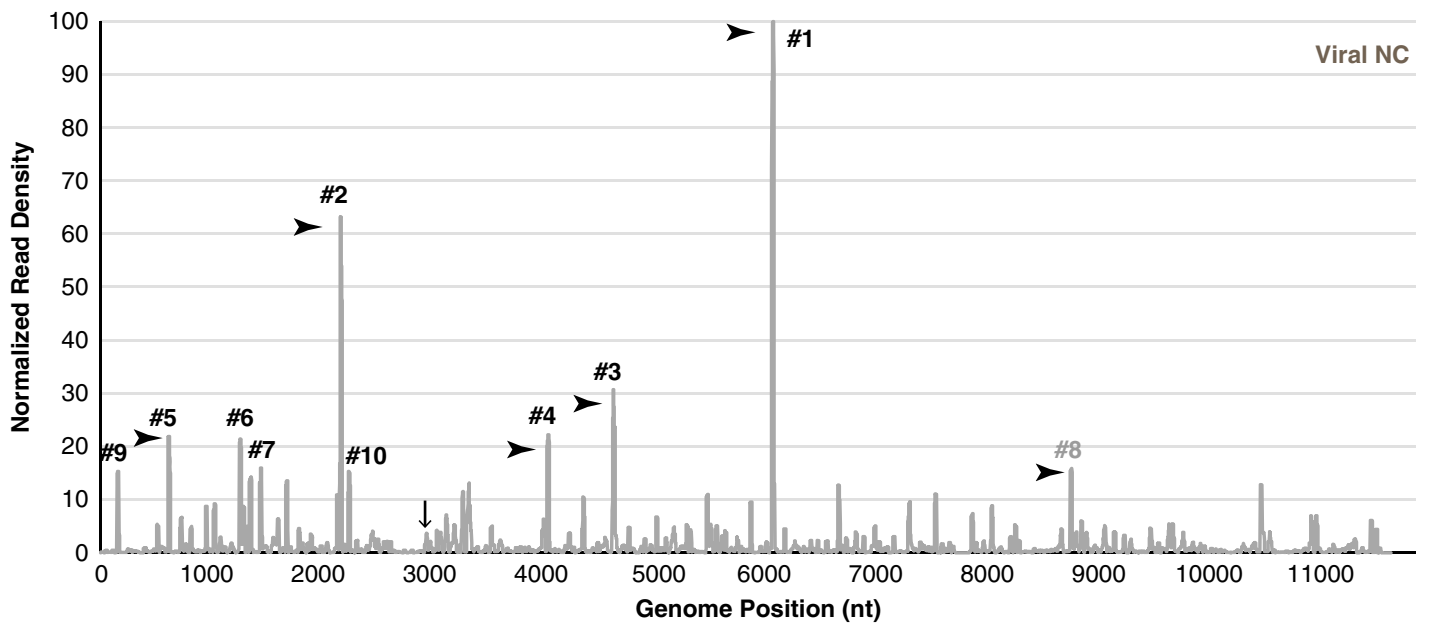

e

NA-specific

sgRNA-overlap
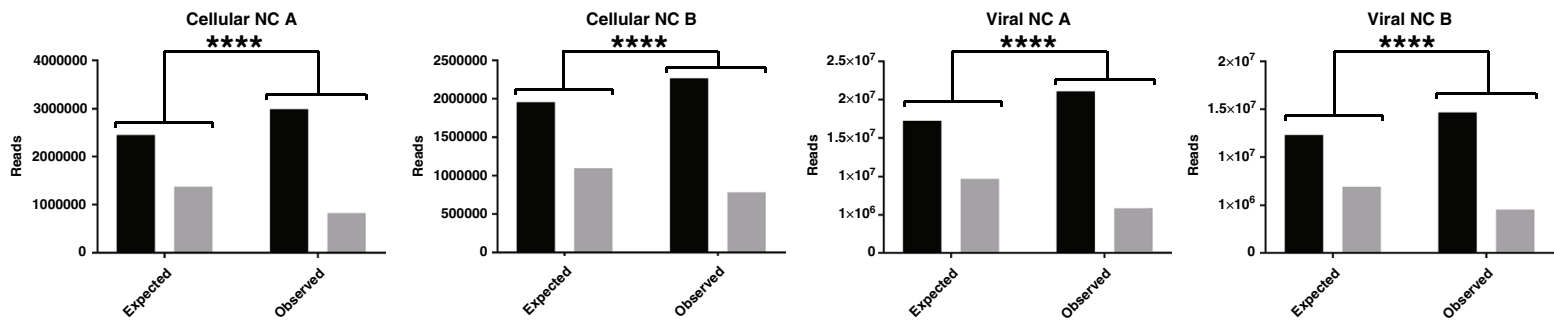

(1:1 Cp:RNA) or in the presence of 10mer DNA (1:20:1 Cp:10mer: RNA) showed a single peak centered toward the middle of the gradient (Fig. 6c), consistent with previous sucrose gradient sedimentation analyses ${ }^{21}$. Thus, Cp robustly assembles into CLPs with site \#1 RNA even in the presence of an excess of electrostatic competitor nucleic acid.

We then tested the effect of mutation of site \#1's sequence on CLP assembly (Fig. 6d). Cp robustly assembled into CLPs with site \#1 RNA in the presence of $10 \mathrm{mer}$ at physiological salt concentration (Fig. 6d, $150 \mathrm{mM} \mathrm{NaCl}$ panel). However, under the same conditions CLP formation with the mutant RNA was impaired (Fig. 6d, $150 \mathrm{mM} \mathrm{NaCl}$ panel). This decrease in CLP 
Fig. 5 Changes in Cp-gRNA interactions triggered by virus budding. a Schematic of the different $\mathrm{Cp}$ and NC populations present during virus assembly. Cp (green), gRNA (black), E2 (pale blue), and E1 (colored by domain: dlll blue, dll yellow, and dl red). b Autoradiogram of Cp-RNA crosslinked adducts as in Fig. 1e, but for purified cellular NC and viral NC (representative of $n=2$ biological replicates). c, d Normalized read densities as in Fig. 2a, but for cellular $\mathrm{NC}(\mathbf{c})$ and viral NC (d). Reads were normalized to the nucleotide position with the highest read frequency within each library, which were read values of 10,143 and 5,855 for cellular NC and 140,546 and 88,226 for viral NC, before respectively averaging. Downward-facing arrow indicates Cp's specific binding site on the PS, and numbered sites represent the top 10 sites bound for each respective sample type. Arrowheads indicate examples of $\mathrm{Cp}$ binding sites maintained throughout virus assembly, and leftward-pointed arrows indicate examples of distinct $\mathrm{Cp}$ interactions enriched only in the cellular NC assembly state. $\mathbf{e} \chi^{2}$ analysis test as in Fig. 3a, but for cellular NC and viral NC biological replicate libraries (a, b). Two-sided, df $=1$, ${ }^{\star \star \star \star} p<0.0001$. See also Supplementary Fig. 2 and Supplementary Data 2.

formation was more apparent when the assembly reaction's salt concentration was raised to shield non-specific electrostatic interactions. Site \#1 induced some CLP formation even at 300 $\mathrm{mM} \mathrm{NaCl}$, while the mutant only assembled CLPs at $150 \mathrm{mM}$ $\mathrm{NaCl}$ (Fig. 6d). Thus, site \#1 RNA specifically promotes CLP assembly in vitro even when general $\mathrm{Cp}$ electrostatic interactions are masked, suggesting that site \#1 on the gRNA may also act to promote NC assembly in infected cells.

\section{Discussion}

We employed PAR-CLIP and biotin-based Cp retrieval as an experimental approach uniquely suited to define the interactions of the alphavirus $\mathrm{Cp}$ with the genomic RNA. Our approach identified a set of high-confidence $\mathrm{Cp}$ binding sites on the SFV gRNA, including an RNA sequence that specifically bound and assembled with $\mathrm{Cp}$ both in infected cells and in vitro. The $\mathrm{Cp}$ binding sites were preferentially distributed on the first $2 / 3$ of the gRNA, suggesting a molecular rationale for the previously observed selective packaging of gRNA over sgRNA ${ }^{9-11}$. An overall preferential binding to gRNA-specific sequences was maintained across different NC assembly states. Such continuous engagement with gRNA-specific sequences can promote gRNA packaging and high infectivity.

A previous study used CLIP to identify SINV Cp interactions with the gRNA in infected cells ${ }^{12}$. Comparison with our results showed that the SINV and SFV Cp top binding sites do not overlap, and that SINV Cp's major binding sites are located within the last $\sim 1 / 3$ rd of the gRNA sequence, overlapping with the sgRNA. Mutation of the SINV Cp binding sites does not affect gRNA packaging or NC assembly, but instead affects the stability of the incoming gRNA. These differences may reflect biological differences between SINV and SFV, but could also be due to differences in experimental approaches.

Alphavirus gRNA packaging signals were originally identified through analysis of DI-RNAs ${ }^{19,20,41}$. Elegant studies showed that appending the SINV PS onto reporter or helper RNAs could increase their packaging in replicon systems and their direct binding to $\mathrm{Cp}$ in vitro, although the increase was at most 5fold ${ }^{19,42}$. Conversely, SINV or VEEV with mutated PSs are impaired but viable ${ }^{18}$. While our data are the first to show that the alphavirus Cp directly binds the DI-RNA-derived PS in cell culture, we also found that genome packaging within the SFV complex does not require the PS. Instead, our data argue that packaging involves preferential $\mathrm{Cp}$ binding to multiple gRNAspecific sites. Although the relative importance of the PS appears to vary between alphavirus complexes, it seems clear that the PS is not strictly required for gRNA packaging/NC assembly. Given that recent studies indicate that DI-RNAs and defective viral genomes can have multifaceted functions (reviewed in Vignuzzi and Lopez ${ }^{43}$ ), the prior DI-RNA studies may have more complex interpretations than previously suspected.

We found that while mutation of multiple high affinity $\mathrm{Cp}$ binding sites did not significantly affect viral replication or protein expression, it significantly reduced infectious virus production. This correlated with reduced gRNA but elevated sgRNA levels in virus particles. Inhibition of infectious virus production was strongest at early stages of virus assembly, in keeping with increasing $\mathrm{Cp}$ concentrations at later times of infection driving $\mathrm{Cp}$ engagement with mutated binding sites. Based on our results we propose a model for selective genome packaging via Cp's interactions with multiple sites selectively located on the gRNA (Fig. 7). At early stages of virus assembly, gRNA levels are high but intracellular Cp levels are relatively low, leading to preferential $\mathrm{Cp}$ binding to high affinity sites on the gRNA (Fig. 7ia vs. ib). This stage may involve Cp dimers ${ }^{22}$, and could be represented by a proposed $90 \mathrm{~S}$ NC assembly intermediate (not shown), which has a lower Cp:gRNA ratio than cellular $\mathrm{NC}^{44-46}$. For some alphaviruses such as SINV and VEEV, this early stage may involve high affinity binding of Cp to the PS. As $\mathrm{Cp}$ levels accumulate in the cell, $\mathrm{Cp}$ begins engaging with lower affinity sites on the gRNA, enabling compaction of the gRNA molecule through charge neutralization ${ }^{16,38,47}$ (Fig. 7ii). More $\mathrm{Cp}$ would then be recruited until completion of the $\mathrm{NC}^{48}$ (Fig. 7iii). E2-Cp interactions promote budding of the NC to produce the enveloped viral particle, a process that results in changes in Cp-RNA interactions (Fig. 7iv). Additional unknown factors may influence genome packaging and NC assembly, including specific subcellular locations or host factors.

From a broader perspective, a multi-site packaging mechanism could be favorable for many RNA viruses given that RNA polymerases generally have low fidelity. Their relatively high mutational frequency produces genetic diversity important for RNA virus adaption to environmental changes, but can also decrease virus fitness ${ }^{49,50}$. A multi-site packaging mechanism could help to ensure correct genome packaging even if deleterious mutations arose in several Cp binding sites. For example, retroviruses such as HIV-1 have high mutation rates and a defined packaging signal known as the $\Psi$ element (reviewed in D'Souza and Summers ${ }^{51}$ ). However, $\Psi$ mutants only modestly decrease ( 3-5-fold) infectious virus production or genome packaging ${ }^{33,52}$. Multi-site packaging mechanisms have been described for RNA viruses such as parechovirus $1^{53}$ and hepatitis $B$ virus ${ }^{54}$, as reviewed in Twarock and Stockley ${ }^{55}$. In addition, multi-site packaging may promote successful co-assembly of Cps and large ssRNAs while decreasing non-productive assembly pathways ${ }^{39}$. Thus, the multisite-based packaging that we describe here for alphaviruses may be a representative of a more general mechanism utilized by many RNA viruses to selectively package their genomes.

Our data represent the first demonstration of a discrete RNA sequence, site \#1, with which alphavirus Cp specifically binds and assembles both in cells and in vitro. While multiple binding sites such as site \#1 were maintained across virus assembly, we also observed discrete differences in Cp:gRNA interactions between the cellular and viral NCs. This could reflect changes induced by different chemical environments, or could be induced by envelope protein association and virus budding. Changes in NC architecture during various stages of virus exit have been previously observed using morphological and biochemical techniques ${ }^{56-59}$. 
a

Site \#1

Mutant

ACAUGGUUGACGGGUCGGAUAGUUGCUUG AUAUGGUAGAUGGAAGCGACUCAUGUCUC

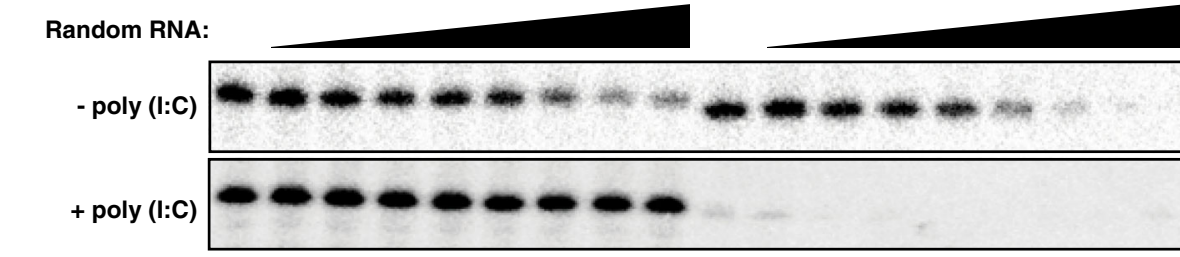

b
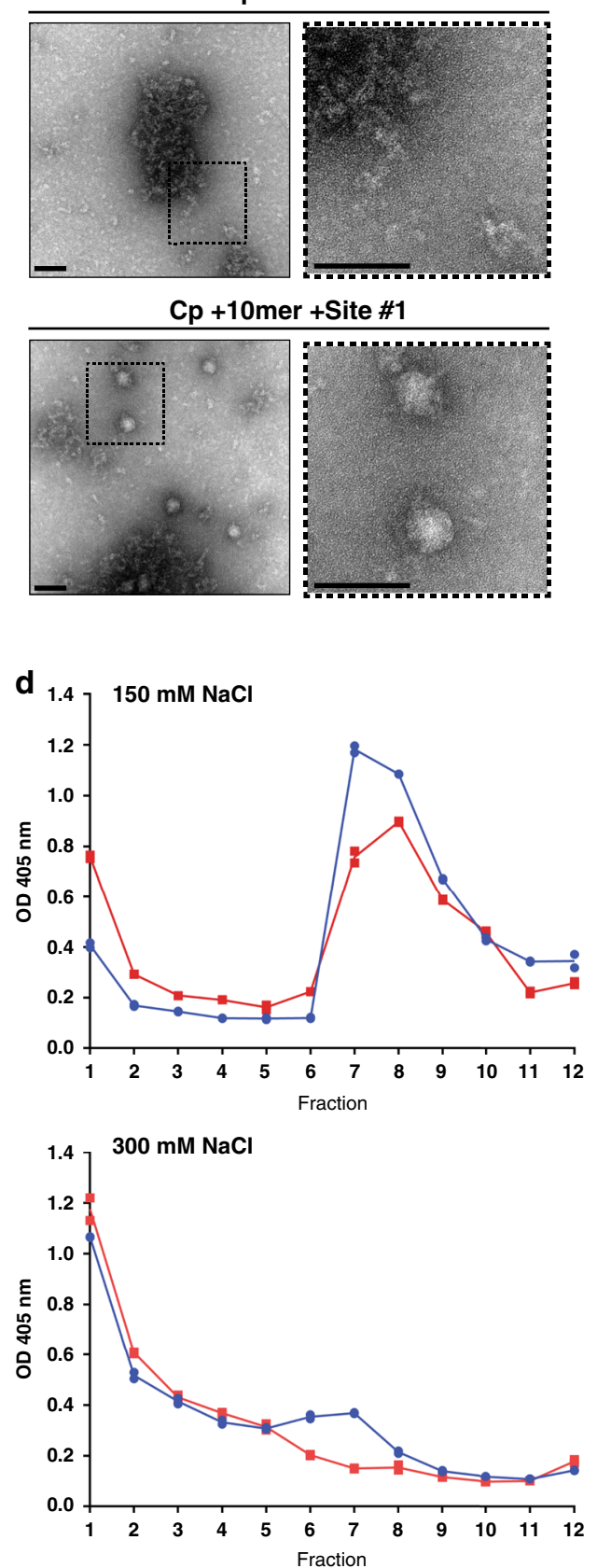
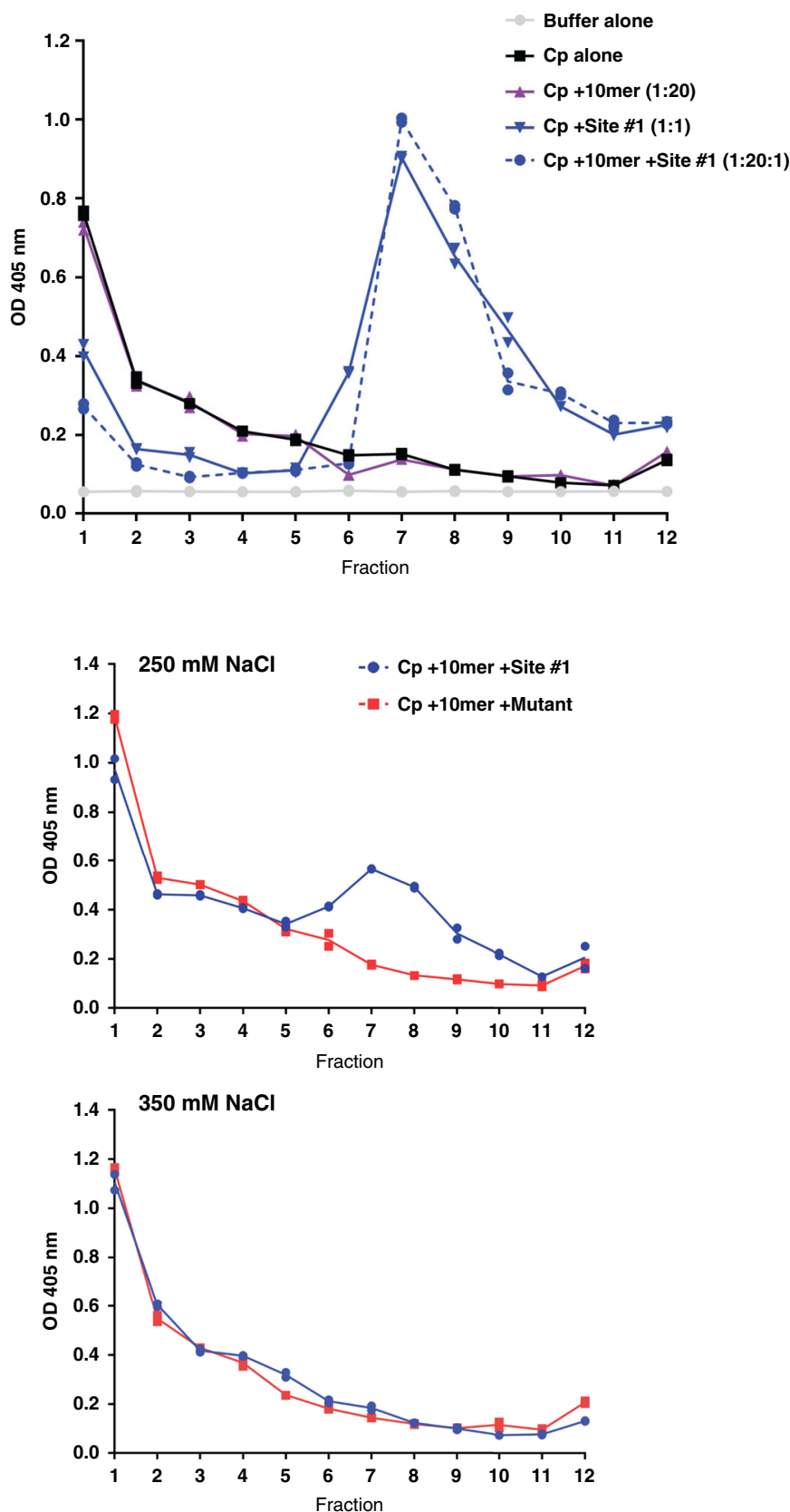

potential importance of such changes in virus Cp-RNA interactions during exit.

\section{Methods}

Note, the included Source Data files contain full images of gels and spreadsheets of data for graphs, etc. interacting face of the NC shell to the inner RNA core of the virus particle. Further functional analyses are needed to determine the
Our data thus suggest that such changes in the NC structure also significantly affect Cp-gRNA binding. If E2 interactions/budding affect Cp-gRNA interactions, this would suggest that changes in $\mathrm{NC}$ architecture are propagated from the outer, envelope-protein 
Fig. 6 Cp specifically binds and assembles with site \#1 in vitro. a Upper panel: $333 \mathrm{nM}$ 32P-labeled site \#1 (left) or mutant (right; mutated residues highlighted in red) RNAs were bound to 13.3 pmole Cp immobilized on Streptactin beads in a 30- $\mu \mathrm{L}$ reaction. Increasing concentrations (4-1000 nM) of random RNA were added as a competitor. Bound RNAs were extracted and subjected to Urea-PAGE followed by autoradiography. Lower panel: as above, but immobilized $\mathrm{Cp}$ was first preincubated with $20 \mu \mathrm{g} / \mathrm{mL}$ of poly $(\mathrm{I}: \mathrm{C})$ to shield non-specific electrostatic interactions. Results are representative of two independent experiments. $\mathbf{b}$ Negative stain EM of CLP assembly reactions of $\mathrm{Cp}+10$ mer or Cp +10 mer + Site \#1 RNA. Dashed boxes in the right panel are $\times 3$ magnification of the corresponding left panel. Scale bar is $80 \mathrm{~nm}$. Images represent one experiment. c CLP assembly reactions were incubated for $30 \mathrm{~min}$ at $25^{\circ} \mathrm{C}(150 \mathrm{mM} \mathrm{NaCl})$ and then analyzed by sucrose gradient sedimentation. $\mathrm{Cp}$ from aliquots of fractions was detected by ELISA. Individual data points from technical duplicates are plotted and the line represents their mean. Fraction 1 is the top of the gradient. Results are representative of $n=4$ independent experiments for $\mathrm{Cp}$ alone, $\mathrm{Cp}+10$ mer (1:20), Cp + Site \#1 (1:1), and Cp +10mer + Site \#1 (1:20:1), and $n=2$ independent experiments for Buffer alone. d As in c, except the indicated salt concentrations were used in CLP assembly reactions comparing CLP assembly with site \#1 or mutant RNAs. Individual data points from technical duplicates are plotted and the line represents their mean. Results are representative of $n=4$ independent experiments for $250 \mathrm{mM} \mathrm{NaCl}$, and $n=2$ independent experiments for 150, 300, and $350 \mathrm{mM} \mathrm{NaCl}$. See also Supplementary Fig. 5 .

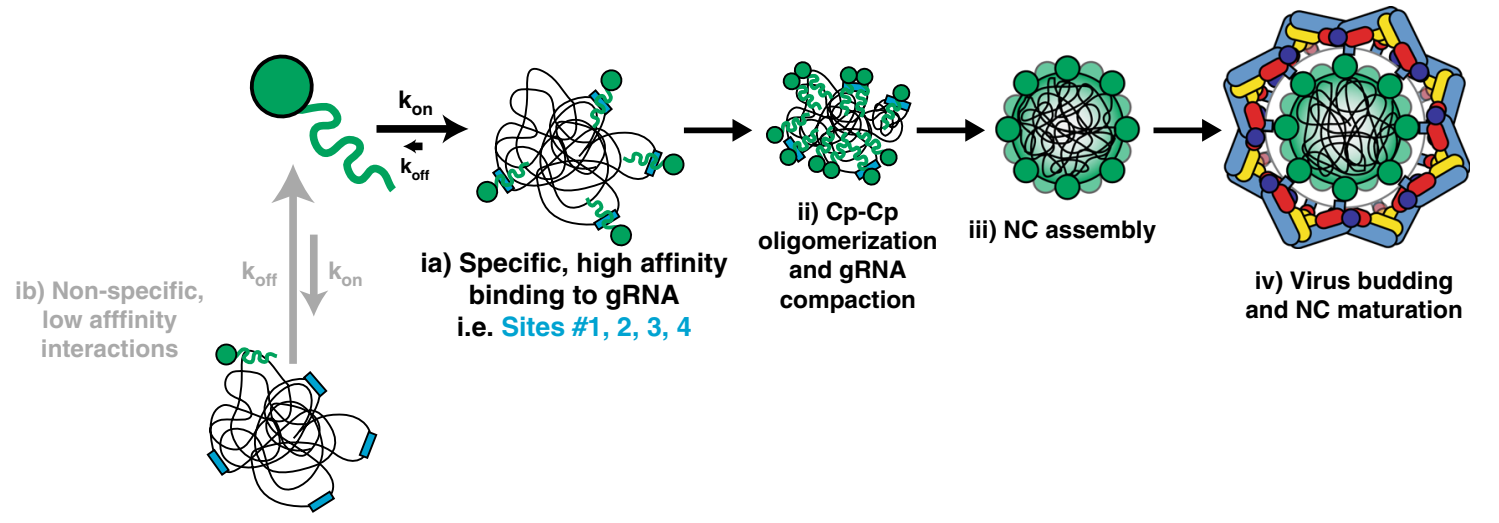

Fig. 7 A multi-site genome packaging model for alphaviruses. (ia) $\mathrm{Cp}$ (green) specifically interacts with high affinity sites (blue rectangles) on the gRNA (black lines). Such high affinity interactions (low Kd) may facilitate longer dwell times for $\mathrm{Cp}$ on the gRNA (slow $k_{\text {off }}$ ), resulting in stable binding. (ib) $\mathrm{Cp}$ can also non-specifically interact with low affinity sites (high Kd) on the gRNA or other RNAs such as the sgRNA or cellular RNAs (not shown), but such low affinity interactions may lead to short dwell times for $\mathrm{Cp}$ on those RNAs (fast $k_{\text {off }}$ ) and less stable binding. (ii) As $\mathrm{Cp}$ levels increase in the cell, $\mathrm{Cp}$ begins binding multiple high affinity sites on the gRNA and stays stably bound (low Kd). This may enable further $\mathrm{Cp}$ recruitment, including $\mathrm{Cp}$ dimer formation, and lead to $\mathrm{Cp}-\mathrm{Cp}$ oligomerization and gRNA compaction. (iii) NC assembly is completed, resulting in 1 copy of the gRNA surrounded by a shell comprised of 240 copies of $\mathrm{Cp}$ arranged with icosahedral symmetry. (iv) Virus assembly and budding occur at the plasma membrane where $\mathrm{Cp}$ directly binds the cytoplasmic domain of the E2 envelope protein (coloring as in Fig. 5a). Virus assembly and budding cause discrete changes in Cp:gRNA interactions within the viral NC.

Cells. Baby hamster kidney (BHK-21) cells (gift from Dr. Ari Helenius) were cultured in Dulbecco's modified Eagle's medium (DMEM, HyClone) containing 4 $\mathrm{mM}$ L-glutamine, $100 \mathrm{U}$ penicillin/mL, $100 \mu \mathrm{g}$ streptomycin/mL, 10\% tryptose phosphate broth, and $5 \%$ FBS at $37^{\circ} \mathrm{C}$. Vero cells (ATCC; gift from Dr. Kartik Chandran) were cultured in DMEM containing $4 \mathrm{mM}$ L-glutamine, $100 \mathrm{U}$ penicillin/mL, $100 \mu \mathrm{g}$ streptomycin $/ \mathrm{mL}$, and $10 \%$ FBS at $37^{\circ} \mathrm{C}$. Vero cells stably expressing HA-BirA were generated by transfecting cells with the expression plasmid described below using Lipofectamine 2000 (Invitrogen). $48 \mathrm{~h}$ post-transfection, cells were split to $25 \%$ confluency and selected with $1 \mathrm{mg} \mathrm{G} 418 / \mathrm{mL}$ (SigmaAldrich) for 2 weeks. G418-resistant cells were then seeded sparsely and individual clones isolated using cloning cylinders (Corning). Clones were screened for their ability to biotinylate Cp-mAVI by western blot and fluorescence microscopy using streptavidin probes. Cell lines were authenticated by morphologic evaluation and were checked for mycoplasma contamination (MycoAlert ${ }^{\mathrm{TM}}$ PLUS Mycoplasma Detection Kit, Lonza).

Antibodies. The following antibodies and SA probes were used for western blots and immunofluorescence as indicated: monoclonal anti- $\beta$ tubulin (E7; WB-1:3000; Developmental Studies Hybridoma Bank), rabbit polyclonal anti- $\alpha$ tubulin (WB1:5000; Abcam, 18251), rabbit polyclonal anti-E2/E1 (WB-1:4000 and IF-1:500) ${ }^{60}$, monoclonal anti-Cp (42-1; WB-1:4000 and IF-1:500) ${ }^{61}$, monoclonal anti-E2 (E2-1; WB-1:2 dilution of the clarified hybridoma supernatant $)^{62}$, Streptavidin Alexa Fluor 680 Conjugate (WB-1:10,000; ThermoFisher Scientific, S32358), and Streptavidin Alexa Fluor 568 Conjugate (IF-1:700; ThermoFisher Scientific, S11226). Rabbit polyclonal anti-nsP2 (WB-1:3000) was a gift from Dr. Andres Merits. Rabbit polyclonal anti-Cp was generated (Covance) against the C-terminal domain of SFV $\mathrm{Cp}$ (residues 119-267) expressed and purified as described below for full-length $\mathrm{Cp}$. Specificity was confirmed by western blot (1:4000 dilution), immunofluorescence (1:500 dilution) of uninfected vs. infected cells, and co-staining with monoclonal antibody $42-1$. The following secondary antibodies were used: goat-anti-rabbit IgG AP (ELISA-1:1000; Southern Biotech, 4049-04), goat-anti-rabbit IgG DyLight 800 (WB-1:10000; ThermoFisher Scientific, SA5-35571), goat-anti-mouse IgG Alexa
Fluor 680 (WB-1:10,000; ThermoFisher Scientific, A-21057), goat-anti-rabbit IgG Alexa Fluor 680 (WB-1:10,000; ThermoFisher Scientific, A-21076), goat-anti-mouse IgG Alexa Fluor Plus 800 (WB-1:10,000; ThermoFisher Scientific, A32730), goatanti-rabbit IgG Alexa Fluor 405 (IF-1:700; ThermoFisher Scientific, A-31556), and goat-anti-mouse IgG Alexa Fluor 488 (IF-1:700; ThermoFisher Scientific, A1-11001).

Viruses, mutants, and plasmids. A minimal biotin acceptor motif (mAVI tag; LNDIFEAQKIEWH $)^{30,31}$ was inserted after Cp residue 95 by overlapping PCR using restriction sites $\mathrm{XbaI}$ and NsiI in the SFV infectious clone pSP6-SFV4 ${ }^{63}$. Overlapping PCR primers: 5'-CTGAACGACATCTTCGAGGCCCAGAAAATCG AATGGCACaagcaagccgacaag- $3^{\prime}$ and $5^{\prime}$-GTGCCATTCGATTTTCTGGGCCTCG AAGATGTCGTTCAGgtctttcttctttgctgc- $3^{\prime}$. Individual Cp binding site mutants were constructed by overlapping PCR and using the nearest unique restriction sites present in pSP6-SFV4. Double and Quintuple Cp binding site mutants were constructed by restriction digest and subcloning from individual mutants. The 17site mutant, Full PS mutant, and 17-site + Full PS mutant were custom synthesized (Epoch Life Science, Inc), and subcloned by restriction digest into pSP6-SFV4. The Chikungunya Full PS mutant was custom synthesized (Epoch Life Science, Inc) and subcloned by restriction digest into the pSinRep5-181/25 Chikungunya infectious clone (kindly provided by Dr. Terrence Dermody ${ }^{64}$ ). All infectious clone mutants were verified by sequencing the entire region affected by the cloning approach (Genewiz, South Plainfield, NJ) and analyzed by restriction digest to check for plasmid rearrangements. A humanized BirA construct was provided by Vasily Ogryzko ${ }^{65}$ and subcloned into pcDNA3.1+ with an N-terminal HA-tag added by PCR.

Virus stocks. SFV WT and mAVI virus stocks were generated by electroporating BHK cells with in vitro transcribed (IVT) viral RNA and collecting the cell media at $24 \mathrm{~h}$ post-electroporation ${ }^{63}$. Virus-containing media were clarified by centrifugation at $10,621 \times \mathrm{g} 4{ }^{\circ} \mathrm{C}$ for $10 \mathrm{~min}$, and $10 \mathrm{mM}$ HEPES $\mathrm{pH} 8.0$ was added to the 
supernatant before aliquoting and freezing. Virus stocks for growth comparisons of SFV WT, Full PS mutant, and the indicated Cp binding site mutants were generated the same way except that the cell media were collected at $8 \mathrm{~h}$ postelectroporation. CHIKV WT and Full PS mutant stocks were generated as above except that the cell media were harvested at $22 \mathrm{~h}$ post-electroporation. All virus stocks were titered in two independent experiments by plaque assay on BHK cells.

Virus growth curves. Growth curves were performed on Vero cells infected at the indicated multiplicity of infection (MOI) for $1.5-2 \mathrm{~h}$ at $37^{\circ} \mathrm{C}$. At the indicated time points, the virus-containing media were collected, clarified, aliquoted, and frozen at $-80^{\circ} \mathrm{C}$. Aliquots were titered via plaque assay on BHK cells.

Cell lysis and western blot. Vero parental or Vero+BirA cell lines were infected at an MOI $=10$ for $1.5 \mathrm{~h}$ at $37^{\circ} \mathrm{C}$ before transfer into fresh medium containing $50 \mu \mathrm{M}$ biotin. At the indicated time points, the cells were washed and lysed with lysis buffer [50 mM Tris-Cl pH 7.4, $100 \mathrm{mM} \mathrm{NaCl}, 1 \%$ Triton-X-100, 1 mM EDTA, $6 \mathrm{mM} \mathrm{NaPPi}$ (to inhibit post-lysis biotinylation), and an EDTA-free protease inhibitor cocktail (Roche; 1 tablet $/ 10 \mathrm{~mL}$ )] on ice. The lysate was then clarified by centrifugation and the soluble lysate was frozen at $-80^{\circ} \mathrm{C}$. Lysates were subjected to SDS-PAGE followed by transfer to nitrocellulose membranes. Membranes were probed with the indicated primary antibodies and corresponding secondary antibodies conjugated to Alexa Fluor 680 or 800 dyes before imaging on an Odyssey Fc Imaging System (LI-COR Biosciences).

Immunofluorescence. Vero parental or Vero+BirA cells were seeded on coverslips in 24-well plates. Cells were infected at an MOI $=1$ for $1.5 \mathrm{~h}$ at $37^{\circ} \mathrm{C}$, and then fresh medium supplemented with $50 \mu \mathrm{M}$ biotin was added to each well. At $7 \mathrm{hpi}$, the cells were fixed with $4 \%$ paraformaldehyde (Electron Microscopy Sciences) for $20 \mathrm{~min}$ and quenched with $50 \mathrm{mM} \mathrm{NH} \mathrm{H}_{4} \mathrm{Cl}$. The cells were permeabilized with $0.1 \%$ Triton-X-100 for $10 \mathrm{~min}$ and blocked with $0.2 \%$ gelatin. Coverslips were then stained with the indicated primary antibodies followed by the corresponding secondary antibody conjugated to an Alexa-Fluor dye. Images were acquired on a Zeiss Axiovert $200 \mathrm{M}$ microscope and processed using ImageJ.

Transmission electron microscopy. Vero parental or Vero+BirA cell lines were seeded in $35 \mathrm{~mm}$ plates and infected at an MOI $=10$ for $1.5 \mathrm{~h}$ at $37^{\circ} \mathrm{C}$ before transfer into $1.2 \mathrm{~mL}$ fresh medium containing $50 \mu \mathrm{M}$ biotin. At $7.5 \mathrm{hpi}$, the cells were washed once with serum-free medium and then fixed with $2.5 \%$ glutaraldehyde and 2\% paraformaldehyde in $0.1 \mathrm{M}$ sodium cacodylate buffer for $30 \mathrm{~min}$ at room temperature. The Einstein Analytical Imaging Facility then processed the samples by postfixing with $1 \%$ osmium tetroxide and $2 \%$ uranyl acetate, dehydrating with ethanol, and lifting the monolayer from the dish by propylene oxide. The samples were pelleted and embedded, and thin sections were stained with uranyl acetate followed by lead citrate. Images were taken on a JEOL 1200EX electron microscope at $80 \mathrm{kV}$ and assembled using Adobe Photoshop software. For negative stain analysis of CLP assembly reactions, $5 \mu \mathrm{L}$ of the $100 \mu \mathrm{L}$ reaction was applied to glow-discharged carbon-coated copper grids and stained with $1 \% \mathrm{v} / \mathrm{v}$ uranyl acetate. Grids were air dried and then viewed on a FEI Tecnai 20 electron microscope at $120 \mathrm{kV}$.

Specific infectivity. Vero parental and Vero+BirA cells were infected at an $\mathrm{MOI}=$ 10 for $1.5 \mathrm{~h}$ at $37^{\circ} \mathrm{C}$ before supplementing with fresh medium containing $50 \mu \mathrm{M}$ biotin. At $7.5 \mathrm{hpi}$, the virus-containing media were collected and clarified by centrifugation. An aliquot was taken from the clarified supernatant for titering by plaque assay to determine infectious particle number. The remaining supernatant was layered over a $20 \%$ sucrose cushion and centrifuged at 35,000 rpm (Beckman Coulter SW41 rotor) for $3 \mathrm{~h}$ at $4{ }^{\circ} \mathrm{C}$. The pelleted virus was resuspended in TN buffer [50 mM Tris-Cl pH 7.4, $100 \mathrm{mM} \mathrm{NaCl}$, and a protease inhibitor cocktail (Roche; 1 tablet $/ 10 \mathrm{~mL}$ )] on ice overnight. Virus suspensions were serially diluted, boiled in SDS sample buffer, and subjected to SDS-PAGE followed by western blot using an E2-specific monoclonal antibody. E2 signal was imaged and quantified using the Odyssey Fc Imaging System (LI-COR Biosciences) to determine particle number. The specific infectivity was calculated as a ratio of the infectious particle number (plaque forming units) to the E2 signal (total particle number) ${ }^{66}$. Note that we determined particle number by protein rather than gRNA to assess possible defects in gRNA packaging, which could be missed if the particle number was determined by gRNA quantitation.

PAR-CLIP. PAR-CLIP on SFV Cp was performed based on published methods ${ }^{67-70}$. Total cellular Cp population: Vero+BirA cells were counted and seeded in $35 \mathrm{~mm}$ dishes $24 \mathrm{~h}$ before infection. The cells were infected with SFV mAVI at an MOI $=$ 10 for $1.5 \mathrm{~h}$, washed three times, and then placed into media supplemented with $50 \mu \mathrm{M}$ biotin and $100 \mu \mathrm{M} 4$-thiouridine. At $7 \mathrm{hpi}$, the cells were UV-irradiated with $368 \mathrm{~nm}$ light at $0.15 \mathrm{~J} / \mathrm{cm}^{2}$, or control cells were mock UV-irradiated. Cells were then placed on ice, washed three times with PBS, and then lysed with lysis buffer (50 mM Tris-HCL pH 7.4, $100 \mathrm{mM} \mathrm{NaCl}, 1 \%$ NP-40, 1 mM EDTA, $6 \mathrm{mM} \mathrm{NaPPi}$, $0.5 \mathrm{mM}$ DTT, and a complete protease inhibitor tablet (Roche; 1 tablet $/ 10 \mathrm{~mL}$ )) for $10 \mathrm{~min}$ with gentle rocking. The lysate was clarified by centrifugation at $13,000 \mathrm{rpm}$
$4{ }^{\circ} \mathrm{C}$ for $10 \mathrm{~min}$. The soluble lysate was then treated with RNaseT1 $(1 \mathrm{U} / \mu \mathrm{L})$ for $15 \mathrm{~min}$ at room temperature and then placed on ice for $5 \mathrm{~min}$. The samples were then frozen at $-80^{\circ} \mathrm{C}$ and a $5 \%$ input control was taken at this step to compare $\mathrm{Cp}$ expression levels by western blot (Supplementary Fig. 2c). Streptavidin Dynabeads (SA-DB; Dynabeads MyOne Streptavidin C1, ThermoFisher Scientific) were equilibrated with PBS while samples thawed on ice. A final concentration of $0.5 \%$ SDS was added to the samples, mixed, and then incubated with the SA-DB by rocking at $4{ }^{\circ} \mathrm{C}$ for $3 \mathrm{~h}$. The SA-DB were then washed twice with RIPA buffer supplemented with high salt (10 mM Tris-Cl pH 7.4, $0.5 \mathrm{M} \mathrm{NaCl}, 1 \mathrm{mM}$ EDTA, $1 \%$ NP-40, $1 \%$ sodium deoxycholate, $0.1 \%$ SDS, $0.5 \mathrm{mM}$ DTT, and a complete protease inhibitor tablet (Roche; 1 tablet/10 mL)) for $5 \mathrm{~min}$ per wash, once with PBS, and treated with RNaseT 1 at $1 \mathrm{U} / \mu \mathrm{L}$ for $15 \mathrm{~min}$ at room temperature before cooling on ice for $5 \mathrm{~min}$. The SA-DB were washed again with high salt RIPA buffer for $5 \mathrm{~min}$, then washed twice with dephosphorylation buffer ( $50 \mathrm{mM}$ Tris- $\mathrm{Cl} \mathrm{pH}$ 7.9, $100 \mathrm{mM}$ $\mathrm{NaCl}, 10 \mathrm{mM} \mathrm{MgCl}_{2}$, and $1 \mathrm{mM} \mathrm{DTT}$ ), and treated with calf-intestine phosphatase at $0.5 \mathrm{U} / \mu \mathrm{L}$ for $10 \mathrm{~min}$ at $37^{\circ} \mathrm{C}$. The SA-DB were washed once with lysis buffer, twice with PNK buffer (50 mM Tris-Cl pH 7.5, $50 \mathrm{mM} \mathrm{NaCl}, 10 \mathrm{mM} \mathrm{MgCl}_{2}, 5 \mathrm{mM}$ DTT) lacking DTT, then resuspended in complete PNK buffer. In all, $1 \mathrm{U} / \mu \mathrm{L}$ of T4 PNK and $0.5 \mu \mathrm{Ci} / \mu \mathrm{L}$ of ${ }^{32} \mathrm{P}$-ATP were added to the SA-DB for $30 \mathrm{~min}$ at $37^{\circ} \mathrm{C}$ with gentle mixing every $5 \mathrm{~min}$. After $30 \mathrm{~min}, 100 \mu \mathrm{M}$ cold ATP was added for $5 \mathrm{~min}$ at $37^{\circ} \mathrm{C}$. The SA-DB were washed five times with PNK buffer without DTT and then once with PBS. Samples were boiled for $5 \mathrm{~min}$ in SDS sample buffer four sequential times to maximize elution before pooling eluates. The eluates were subjected to SDS-PAGE and then transferred to nitrocellulose membranes for autoradiography. The film was overlaid on top of the membrane and the corresponding CpxRNA crosslinked adducts were excised from the membrane. The membrane was treated with proteinase $\mathrm{K}$ for 90 minutes at $55^{\circ} \mathrm{C}$, and the RNA was extracted with phenol-chloroform and precipitated.

Cellular and viral NCs: PAR-CLIP on cellular and viral NCs was performed as described above, but with the following differences. Two $10 \mathrm{~cm}$ plates of Vero + BirA cells were infected with SFV mAVI at an MOI $=10$. At $7.5 \mathrm{hpi}$, the viruscontaining media were collected and clarified by centrifugation at $10,000 \mathrm{rpm}$ for $5 \mathrm{~min}$ at $4^{\circ} \mathrm{C}$. The virus was then purified over a $20 \%$ sucrose cushion by centrifugation at $35,000 \mathrm{rpm}$ (Beckman Coulter SW41 rotor) for $3 \mathrm{~h}$ at $4{ }^{\circ} \mathrm{C}$. The pellet was resuspended on ice for $3 \mathrm{~h}$ in TN buffer and then transferred to a $35-\mathrm{mm}$ dish. The virus was UV irradiated or mock irradiated with $368 \mathrm{~nm}$ light at $0.15 \mathrm{~J} /$ $\mathrm{cm}^{2}$, and then lysed in lysis buffer, denatured with $0.5 \% \mathrm{SDS}$, and processed as described under Total cellular $\mathrm{Cp}$ population. The cells from the same plates were washed with PBS and then UV-irradiated with $368 \mathrm{~nm}$ light at $0.15 \mathrm{~J} / \mathrm{cm}^{2}$ or mockirradiated. Cells were pelleted, lysed in lysis buffer, clarified by centrifugation at $13,000 \mathrm{rpm}$ for $10 \mathrm{~min}$ at $4^{\circ} \mathrm{C}$, and then treated with $25 \mathrm{mM}$ EDTA for $20 \mathrm{~min}$ to dissociate polysomes. Samples were loaded onto $7.5-20 \%$ (wt/wt) sucrose gradients in TN buffer $+2 \mathrm{mM}$ EDTA and $0.1 \% \mathrm{NP}-40$, and centrifuged at $41,000 \mathrm{rpm}$ (Beckman Coulter SW41 rotor) for $2 \mathrm{~h}$ at $4{ }^{\circ} \mathrm{C}$. In total, $1 \mathrm{~mL}$ fractions were collected and aliquots were analyzed by SDS-PAGE followed by western blot using an anti-Cp antibody to identify the NC fractions. The NC fractions were pooled, denatured with $0.5 \%$ SDS, retrieved with SA-DB, and processed as described under Total cellular Cp population.

cDNA library construction and sequencing. The extracted RNA was ligated to the $3^{\prime}$ adenylated adapter using Rnl2(1-249)K227Q ligase at $4^{\circ} \mathrm{C}$ overnight. A size marker mix containing synthetic 19- and 35-nt-long RNAs was used as a positive control for all ligation steps. After ligation, the reaction was denatured at $90^{\circ} \mathrm{C}$ for $1 \mathrm{~min}$ and electrophoresed on a $15 \%$ denaturing Urea-PAGE. The gel was then exposed to a phosphoimager screen for an hour at $-20^{\circ} \mathrm{C}$. The image was printed to its original size and aligned on to the gel where the successfully ligated product was excised, shredded, and incubated with $0.3 \mathrm{M} \mathrm{NaCl}$ at $60^{\circ} \mathrm{C}$ for $45 \mathrm{~min}$. The sample was filtered and then the RNA was precipitated in ethanol at $-20^{\circ} \mathrm{C}$ for at least an hour. The precipitated RNA was pelleted and dissolved in water. The $5^{\prime}$ adapter was ligated to the sample using Rnll for $1 \mathrm{~h}$ at $37^{\circ} \mathrm{C}$. The sample was then processed the same way as the $3^{\prime}$ adapter ligation reaction. The ligated sample was reverse transcribed to make cDNA using Superscript III reverse transcriptase for $2 \mathrm{~h}$ at $50{ }^{\circ} \mathrm{C}$. A pilot PCR was performed to optimize PCR cycle number to prevent over amplification of the library. This entailed taking a $10-\mu \mathrm{L}$ aliquot from the pilot PCR after every three cycles between cycles 12 and 30 for agarose gel analysis. The lowest PCR cycle number to generate a visible PCR product by ethidium-bromide staining was used for the final PCR. The final PCR product was purified away from linker-linker ( $3^{\prime}$ adapters ligated to $5^{\prime}$ adapters) byproducts using a 3\% Pippin Prep (Sage Science) before sequencing on an Illumina MiSeq machine. Oligonucleotides used $\left(5^{\prime}\right.$ to $\left.3^{\prime}\right)$ :

RNA PCR Index Primer 9

CAAGCAGAAGACGGCATACGAGATCTGATCGTGACTGGAGTTCCTTGGC ACCCGAGAATTCCA

RNA PCR Index Primer 10

CAAGCAGAAGACGGCATACGAGATAAGCTAGTGACTGGAGTTCCTTGGC ACCCGAGAATTCCA;

RT Primer GCCTTGGCACCCGAGAATTCCA; GG-L;

3' Barcode adapter 29.31 5'-rAppNNTAGCGATGGAATTCTCGGGTGCCAA 
3' Barcode adapter 29.32 5'-rAppNNCTGTAGTGGAATTCTCGGGTGCCAA GG-L;

3' Barcode adapter 29.33 5'-rAppNNTAGTCGTGGAATTCTCGGGTGCCAA GG-L;

$\mathrm{L}=$ aminolinker, $3^{\prime}$-amino modifier $\mathrm{C}$.

RT-qPCR. To quantify viral RNA in infected cells, Vero cells were mock infected or infected with SFV WT or 17 -site mutant at an MOI $=0.01$ for $1.5 \mathrm{~h}$ at $37^{\circ} \mathrm{C}$. At the indicated time points, cell-associated RNA was extracted using Trizol as per the manufacturer's instructions. Total RNA was quantified by Nanodrop and RNA integrity was assessed by $2 \%(\mathrm{v} / \mathrm{v})$ bleach agarose gel electrophoresis ${ }^{71}$ to visualize rRNA. To quantify viral RNA in released particles, Vero cells in two $10 \mathrm{~cm}$ plates were infected as above. At the indicated time points, virus-containing media were collected and clarified by centrifugation. An aliquot was taken from the clarified supernatant for titering via plaque assay to determine infectious particle number. The remaining supernatant was layered over a $20 \%$ sucrose cushion and pelleted (c.f. Methods, Specific Infectivity). Pellets were resuspended in $100 \mu \mathrm{L}$ of $50 \mathrm{mM}$ Tris $\mathrm{pH}$ 7.4, $100 \mathrm{mM} \mathrm{NaCl}, 1 \mathrm{mM}$ EDTA, protease inhibitor (Roche; 1 tablet/10 $\mathrm{mL}), 1 \mathrm{U} / \mu \mathrm{L}$ recombinant Rnasin (RNase inhibitor) before extracting viral RNA using the MagMax viral RNA isolation kit as per the manufacturer's instructions. IVT gRNA was produced from SFV infectious clone plasmid and treated with $10 \mathrm{U}$ of DNase1 (RNase-Free; NEB) for $30 \mathrm{~min}$ at $37^{\circ} \mathrm{C}$ before purification with the RNeasy MiniElute Cleanup kit (Qiagen) as per the manufacturer's instructions. IVT gRNA integrity and purity was assessed by agarose gel analysis and quantified by NanoDrop. cDNA was synthesized from $5 \mathrm{ng}$ of extracted cell-associated RNA, $5 \mathrm{ng}$ of IVT gRNA, or $10 \%$ of pelleted viral RNA using the Verso cDNA synthesis kit and gRNA-specific or [gRNA+sgRNA] reverse primers. qPCR was performed using Power SYBR Green PCR Master Mix (Applied Biosystems) in a ViiA 7 RealTime PCR machine using 384-well plates. cDNA from samples corresponding to three biological replicates were assayed with technical duplicates. A no template control and mock-infected controls were used to ensure viral RNA-specific amplification. Four standard curves consisting of a 10-fold dilution series of IVT gRNA cDNA were used to calculate sample gRNA and [gRNA+sgRNA] copy numbers by relating the threshold cycle values. Cycle conditions were $50{ }^{\circ} \mathrm{C} 2 \mathrm{~min}$, $95^{\circ} \mathrm{C} 10 \mathrm{~min}$, and 45 cycles of $95^{\circ} \mathrm{C} 15 \mathrm{~s}$ and $60^{\circ} \mathrm{C} 1 \mathrm{~min}$, followed by melt curve analysis. Oligonucleotides used $\left(5^{\prime}-3^{\prime}\right)$ : gRNA-specific forward primer CTACGC TACACCAGATGAATACC, gRNA-specific reverse primer GGCTATGTCTGCTC TCTTAACTC, [gRNA + sgRNA] forward primer GCCTCGAACCAACCCTTAAT, and [gRNA + sgRNA] reverse primer CTTCTCTTTAGTGGAGCACTCTG.

Recombinant $\mathrm{Cp}$ expression and purification. SFV $\mathrm{Cp}$ was cloned via PCR into pET29a with an N-terminal double Strep Tag (WSHPQFEK) followed by a glycine + serine linker and a TEV protease cleavage site (2XStrep_GS_TevClvg_Cp) for expression in Rosetta2 cells. Rossetta2 cells were grown at $30^{\circ} \mathrm{C}$ until an $\mathrm{OD}_{600}$ of 1.0 , and protein expression was induced with IPTG at $16^{\circ} \mathrm{C}$ overnight. Cells were harvested and resuspended in binding buffer $(100 \mathrm{mM}$ Tris- $\mathrm{Cl} \mathrm{pH}$ 8.0, $150 \mathrm{mM}$ $\mathrm{NaCl}, 1 \mathrm{mM}$ EDTA, complete protease inhibitor cocktail (Roche; 1 tablet/10 mL) before sonicating on ice. The lysate was clarified by two centrifugation steps. The soluble lysate was then diluted to $10 \mathrm{X}$ the volume in binding buffer supplemented with $1.5 \mathrm{M} \mathrm{NaCl}$, mixed, and placed on ice overnight. The next day, the protein was purified via affinity chromatography using Strep-Tactin sepharose (IBA) and then dialyzed into $50 \mathrm{mM}$ Tris-Cl pH 7.4 and $100 \mathrm{mM} \mathrm{NaCl}$ before concentrating and freezing. Protein purity ( $>99 \%)$ was assessed by SDS-PAGE and Coomassie staining (Supplementary Fig. 5a). The sample's $A_{260} / A_{280}$ ratio showed no RNA or DNA contamination.

In vitro core-like particle assembly. HPLC purified RNA oligos corresponding to site \#1 or its mutant version were purchased from IDT. RNAs were denatured at $90^{\circ} \mathrm{C}$ for $2 \mathrm{~min}$ and chilled on ice for $10 \mathrm{~min}$ before serially diluting to $10 \mu \mathrm{M}$ in $50 \mathrm{mM}$ K-HEPES pH 7.0, $200 \mathrm{mM} \mathrm{KCl}, 10 \mathrm{mM} \mathrm{MgCl}$. Diluted RNAs were incubated at $37^{\circ} \mathrm{C}$ for $30 \mathrm{~min}$ to promote folding before placing back on ice. CLPs were assembled in a $100-\mu \mathrm{L}$ volume in reaction buffer [ $50 \mathrm{mM}$ Tris $\mathrm{pH}$ 7.0, 150 $\mathrm{mM} \mathrm{NaCl}, 5 \mathrm{mM} \mathrm{MgCl} 2,5 \mathrm{mM} \mathrm{KCl}, 0.01 \%(\mathrm{v} / \mathrm{v})$ Tween 20, $1 \mu \mathrm{g} / \mu \mathrm{L}$ BSA, $5 \mathrm{mM}$ DTT, and $0.8 \mathrm{U} / \mu \mathrm{L}$ of recombinant RNasin (RNase inhibitor)]. Cp was diluted (500 $\mathrm{nM}$ final concentration) in reaction buffer before adding 10mer DNA oligo (CCGTTAATGC; $10 \mu \mathrm{M}$ final concentration) or buffer control (50 mM Tris $\mathrm{pH}$ $7.4,100 \mathrm{mM} \mathrm{NaCl}$ ) for $10 \mathrm{~min}$ at RT. Reactions were placed on ice and then each RNA oligo (500 nM final concentration) or buffer control was added. CLP assembly reactions were incubated at $25^{\circ} \mathrm{C}$ for $30 \mathrm{~min}$ before loading onto $15-30 \%$ (wt/wt) sucrose gradients and spinning at $159,599 \times g$ for $43 \mathrm{~min}$ at $4{ }^{\circ} \mathrm{C}$ (Beckman Coulter TLS 55 rotor). Gradients were immediately fractionated and aliquots of each fraction were denatured by boiling in 1\% SDS to ensure antibody access, and then diluted into binding buffer $(50 \mathrm{mM}$ Tris $\mathrm{pH} 8.0,150 \mathrm{mM} \mathrm{NaCl}, 2 \mathrm{mM}$ EDTA, and $1 \% \mathrm{NP}-40)$ to make a mixed detergent micelle $(\sim 0.075 \%$ SDS final concentration). A corresponding standard curve consisting of a 2 -fold dilution series of purified Cp was similarly processed to mimic sucrose, SDS, and boiling conditions (Supplementary Fig. 5b) for every CLP experiment. Samples were bound in duplicate or triplicate to high-capacity streptavidin-coated plates (Pierce) preblocked with $1 \%$ BSA in TBS (25 mM Tris pH 7.2, $150 \mathrm{mM} \mathrm{NaCl}$ ) for $\sim 12-18 \mathrm{~h}$ at $4{ }^{\circ} \mathrm{C}$. Plates were incubated with a polyclonal antibody to $\mathrm{Cp}$ (1:1000 dilution) followed by secondary antibody conjugated to alkaline phosphatase (1:1000 dilution). pNPP substrate was added for $15 \mathrm{~min}$ before measuring absorbance at 405 $\mathrm{nm}$ on a Tecan Infinite F50 plate reader.

In vitro binding assay. In vitro binding assays were performed following the RNA Bind-n-Seq protocol ${ }^{72}$ with the following modifications. Recombinant $\mathrm{Cp}$ was immobilized on Strep-TactinXT coated magnetic beads $(20$ pmole protein $/ \mu$ l bead slurry) in buffer w (100 mM Tris-Cl pH 8.0, $150 \mathrm{mM} \mathrm{NaCl}$, and $1 \mathrm{mM}$ EDTA). Beads were resuspended in RNA-binding buffer $(25 \mathrm{mM}$ Tris $\mathrm{pH} 7.5,150 \mathrm{mM} \mathrm{KCl}$, $3 \mathrm{mM} \mathrm{MgCl}_{2}, 0.01 \%(\mathrm{v} / \mathrm{v})$ Tween $20,1 \mathrm{mg} / \mathrm{mL} \mathrm{BSA}$, and $\left.1 \mathrm{mM} \mathrm{DTT}\right)$. Binding reactions were prepared in 96-well plates. A total of 13.3 pmole of $\mathrm{Cp}$ was immobilized on beads per $30 \mu \mathrm{l}$ reaction and concentrations of $333 \mathrm{nM}$ site \#1 or its mutant along with increasing concentrations of random 29 mer RNA (4-1000 nM) were added in each well with or without $20 \mu \mathrm{g} / \mathrm{ml}$ poly(I:C) preincubation. Binding was performed for 30 minutes at $30^{\circ} \mathrm{C}$ and the plate was then placed on a 96-well magnetic rack. The beads were washed three times with wash buffer $(25 \mathrm{mM}$ Tris $\mathrm{pH} 7.5,150 \mathrm{mM} \mathrm{KCl}, 60 \mu \mathrm{g} / \mathrm{mL}$ BSA, $0.5 \mathrm{mM}$ EDTA, and $0.01 \%(\mathrm{v} / \mathrm{v})$ Tween 20). In all, $40 \mu$ of elution buffer (10 mM Tris pH 7.0, $1 \mathrm{mM}$ EDTA, $1 \%$ SDS) was added to the beads before heating at $70^{\circ} \mathrm{C}$ for $10 \mathrm{~min}$. The supernatant from each well was placed in a new plate and the bound RNA was purified away from Cp by phenol/chloroform isolation. The purified RNA was resolved by $15 \%$ denaturing Urea-PAGE.

PAR-CLIP data analysis. Adapters were removed using Cutadapt $2.4^{73}$ and the remaining sequences were mapped to the SFV4 genome using Bowtie/1.1.1. Only mapped sequences that contained the characteristic $\mathrm{T}$-to- $\mathrm{C}$ mutation were further used. Initial analyses compared sequence reads (uncollapsed reads) vs. unique reads stemming from the barcoded adapters (collapsed reads) to assess PCR duplicates. No significant difference was observed in Cp's top binding sites between the uncollapsed vs. collapsed reads, which was expected because we carefully optimized PCR cycle number during cDNA library construction (as described above). We therefore continued our analyses with the uncollapsed reads. Reads were normalized to the nucleotide position with the highest read frequency within the library, and then biological replicates were averaged and renormalized to produce the final normalized read density. High confidence binding sites were manually defined with the following criteria: the binding site must be at least $10 \mathrm{nt}$ long, have at least one position containing an average normalized read density $\geq 5.0$, and both replicate libraries must have $\geq 250$ reads at that position. Within these binding sites, the $5^{\prime}$ and $3^{\prime}$ ends were generally defined by having $\geq 1.5$ average normalized read density, $\geq 10$ reads per replicate library, and $3^{\prime}$ G-bias due to RNaseT1 digest. Low confidence binding sites were defined as those with at least an average normalized read density $\geq 1.0$ and $\geq 10$ reads for each replicate library.

Statistical analysis. Statistical analyses were performed using GraphPad Prism 7.04 (GraphPad Software). The specific statistical tests used and the number of replicates per experiment are stated in the figure legends. In all graphs, four asterisks indicate a $P$-value of $<0.0001$ and "ns" indicates not significant. Exact $P$ values for all statistical tests are listed in the Source Data.

Reporting summary. Further information on research design is available in the Nature Research Reporting Summary linked to this article.

\section{Data availability}

The PAR-CLIP datasets are publicly available in the NCBI GEO repository under GEO accession number GSE156313. The source PDB file used to create the image in Fig. 1a is publicly available in the RCSB Protein Data Bank as PDB accession number $6 \mathrm{MX} 7$. Source data are provided with this paper.

Received: 1 August 2019; Accepted: 24 August 2020; Published online: 17 September 2020

\section{References}

1. Kuhn, R. J. In Fields Virology. Vol. 1 (eds. D. M. Knipe \& P. M. Howley) Ch 22, 629-650 (Lippincott, Williams and Wilkins, 2013).

2. Weaver, S. C., Winegar, R., Manger, I. D. \& Forrester, N. L. Alphaviruses: population genetics and determinants of emergence. Antivir. Res. 94, 242-257 (2012).

3. Suhrbier, A., Jaffar-Bandjee, M. C. \& Gasque, P. Arthritogenic alphaviruses-an overview. Nat. Rev. Rheumatol. 8, 420-429 (2012).

4. Levi, L. I. \& Vignuzzi, M. Arthritogenic alphaviruses: a worldwide emerging threat? Microorganisms 7, 133 (2019). 
5. Claflin, S. B. \& Webb, C. E. Ross river virus: many vectors and unusual hosts make for an unpredictable pathogen. PLoS Pathog. 11, e1005070-e1005070 (2015).

6. Wengler, G., Wengler, G., Boege, U. \& Wahn, K. Establishment and analysis of a system which allows assembly and disassembly of alphavirus core-like particles under physiological conditions in vitro. Virology 132, 401-412 (1984).

7. Pietila, M. K., Hellstrom, K. \& Ahola, T. Alphavirus polymerase and RNA replication. Virus Res. 234, 44-57 (2017).

8. Brown, R. S., Wan, J. J. \& Kielian, M. The alphavirus exit pathway: what we know and what we wish we knew. Viruses 10, 89 (2018).

9. Rumenapf, T., Strauss, E. G. \& Strauss, J. H. Subgenomic mRNA of Aura alphavirus is packaged into virions. J. Virol. 68, 56-62 (1994).

10. Owen, K. E. \& Kuhn, R. J. Identification of a region in the sindbis virus nucleocapsid protein that is involved in specificity of RNA encapsidation. $J$. Virol. 70, 2757-2763 (1996).

11. Warrier, R., Linger, B. R., Golden, B. L. \& Kuhn, R. J. Role of sindbis virus capsid protein region II in nucleocapsid core assembly and encapsidation of genomic RNA. J. Virol. 82, 4461-4470 (2008).

12. Sokoloski, K. J. et al. Identification of interactions between sindbis virus capsid protein and cytoplasmic vRNA as novel virulence determinants. PLoS Pathog. 13, e1006473 (2017).

13. Garmashova, N., Gorchakov, R., Frolova, E. \& Frolov, I. Sindbis virus nonstructural protein nsP2 is cytotoxic and inhibits cellular transcription. $J$. Virol. 80, 5686-5696 (2006).

14. Akhrymuk, I., Kulemzin, S. V. \& Frolova, E. I. Evasion of the innate immune response: the Old World alphavirus nsP2 protein induces rapid degradation of Rpb1, a catalytic subunit of RNA polymerase II. J. Virol. 86, 7180-7191 (2012).

15. Kim, B. et al. Discovery of widespread host protein interactions with the prereplicated genome of CHIKV using VIR-CLASP. Mol. Cell 78, 624-640.e7 (2020).

16. Mendes, A. \& Kuhn, R. J. Alphavirus nucleocapsid packaging and assembly. Viruses 10, 138 (2018).

17. Levis, R., Weiss, B. G., Tsiang, M., Huang, H. \& Schlesinger, S. Deletion mapping of Sindbis virus DI RNAs derived from cDNAs defines the sequences essential for replication and packaging. Cell 44, 137-145 (1986).

18. Kim, D. Y., Firth, A. E., Atasheva, S., Frolova, E. I. \& Frolov, I. Conservation of a packaging signal and the viral genome RNA packaging mechanism in alphavirus evolution. J. Virol. 85, 8022-8036 (2011).

19. Frolova, E., Frolov, I. \& Schlesinger, S. Packaging signals in alphaviruses. J. Virol. 71, 248-258 (1997).

20. White, C. L., Thomson, M. \& Dimmock, N. J. Deletion analysis of a defective interfering Semliki Forest virus RNA genome defines a region in the $\mathrm{nsP} 2$ sequence that is required for efficient packaging of the genome into virus particles. J. Virol. 72, 4320-4326 (1998).

21. Tellinghuisen, T. L., Hamburger, A. E., Fisher, B. R., Ostendorp, R. \& Kuhn, R. J. In vitro assembly of alphavirus cores by using nucleocapsid protein expressed in Escherichia coli. J. Virol. 73, 5309-5319 (1999).

22. Tellinghuisen, T. L. \& Kuhn, R. J. Nucleic acid-dependent cross-linking of the nucleocapsid protein of Sindbis virus. J. Virol. 74, 4302-4309 (2000).

23. Mukhopadhyay, S., Chipman, P. R., Hong, E. M., Kuhn, R. J. \& Rossmann, M. $\mathrm{G}$. In vitro-assembled alphavirus core-like particles maintain a structure similar to that of nucleocapsid cores in mature virus. J. Virol. 76, 11128-11132 (2002).

24. Wengler, G., Boege, U., Wengler, G., Bischoff, H. \& Wahn, K. The core protein of the alphavirus Sindbis virus assembles into core-like nucleoproteins with the viral genome RNA and with other single-stranded nucleic acids in vitro. Virol 118, 401-410 (1982)

25. Cheng, F. et al. The packaging of different cargo into enveloped viral nanoparticles. Mol. Pharm. 10, 51-58 (2013).

26. Hafner, M. et al. Transcriptome-wide identification of RNA-binding protein and microRNA target sites by PAR-CLIP. Cell 141, 129-141 (2010).

27. Zheng, Y. \& Kielian, M. Imaging of the alphavirus capsid protein during virus replication. J. Virol. 87, 9579-9589 (2013).

28. Howarth, M. et al. A monovalent streptavidin with a single femtomolar biotin binding site. Nat. Methods 3, 267-273 (2006).

29. Howarth, M. \& Ting, A. Y. Imaging proteins in live mammalian cells with biotin ligase and monovalent streptavidin. Nat. Protoc. 3, 534-545 (2008)

30. Schatz, P. J. Use of peptide libraries to map the substrate specificity of a peptide-modifying enzyme: a 13 residue consensus peptide specifies biotinylation in Escherichia coli. Biotechnology 11, 1138-1143 (1993).

31. Beckett, D., Kovaleva, E. \& Schatz, P. J. A minimal peptide substrate in biotin holoenzyme synthetase-catalyzed biotinylation. Protein Sci. 8, 921-929 (1999).

32. Beitzel, B. F., Bakken, R. R., Smith, J. M. \& Schmaljohn, C. S. High-resolution functional mapping of the venezuelan equine encephalitis virus genome by insertional mutagenesis and massively parallel sequencing. PLoS Pathog. 6, e1001146 (2010)
33. Kutluay, S. B. et al. Global changes in the RNA binding specificity of HIV-1 gag regulate virion genesis. Cell 159, 1096-1109 (2014).

34. Benhalevy, D. et al. The human CCHC-type zinc finger nucleic acid-binding protein binds G-rich elements in target mRNA coding sequences and promotes translation. Cell Rep. 18, 2979-2990 (2017).

35. Sauer, M. et al. DHX36 prevents the accumulation of translationally inactive mRNAs with G4-structures in untranslated regions. Nat. Commun. 10, 2421 (2019).

36. Frith, M. C., Saunders, N. F., Kobe, B. \& Bailey, T. L. Discovering sequence motifs with arbitrary insertions and deletions. PLoS Comput. Biol. 4, e1000071 (2008).

37. Zuker, M. Mfold web server for nucleic acid folding and hybridization prediction. Nucleic Acids Res. 31, 3406-3415 (2003).

38. Twarock, R., Bingham, R. J., Dykeman, E. C. \& Stockley, P. G. A modelling paradigm for RNA virus assembly. Curr. Opin. Virol. 31, 74-81 (2018).

39. Dykeman, E. C., Stockley, P. G. \& Twarock, R. Solving a Levinthal's paradox for virus assembly identifies a unique antiviral strategy. Proc. Natl Acad. Sci. USA 111, 5361-5366 (2014).

40. Rayaprolu, V. et al. Length of encapsidated cargo impacts stability and structure of in vitro assembled alphavirus core-like particles. J. Phys. Condens. Matter. Inst. Phys. J. 29, 484003 (2017).

41. Jalanko, A. \& Soderlund, H. The repeated regions of Semliki Forest virus defective-inferfering RNA interferes with the encapsidation process of the standard virus. Virol 141, 257-266 (1985).

42. Weiss, B., Nitschko, H., Ghattas, I., Wright, R. \& Schlesinger, S. Evidence for specificity in the encapsidation of Sindbis virus RNAs. J. Virol. 63, 5310-5318 (1989).

43. Vignuzzi, M. \& Lopez, C. B. Defective viral genomes are key drivers of the virus-host interaction. Nat. Microbiol. 4, 1075-1087 (2019).

44. Ulmanen, I. Assembly of Semliki Forest virus nucleocapsid: detection of a precursor in infected cells. J. Gen. Virol. 41, 353-365 (1978).

45. Forsell, K., Griffiths, G. \& Garoff, H. Preformed cytoplasmic nucleocapsids are not necessary for alphavirus budding. EMBO J. 15, 6495-6505 (1996).

46. Skoging-Nyberg, U. \& Liljestrom, P. M-X-I motif of semliki forest virus capsid protein affects nucleocapsid assembly. J. Virol. 75, 4625-4632 (2001).

47. Borodavka, A., Tuma, R. \& Stockley, P. G. Evidence that viral RNAs have evolved for efficient, two-stage packaging. Proc. Natl Acad. Sci. USA 109, 15769-15774 (2012)

48. Soderlund, H. Kinetics of formation of the Semliki Forest virus nucleocapsid Intervirology 1, 354-361 (1973)

49. Dolan, P. T., Whitfield, Z. J. \& Andino, R. Mechanisms and concepts in RNA virus population dynamics and evolution. Annu. Rev. Virol. 5, 69-92 (2018).

50. Pfeiffer, J. K. \& Kirkegaard, K. Increased fidelity reduces poliovirus fitness and virulence under selective pressure in mice. PLoS Pathog. 1, e11 (2005).

51. D'Souza, V. \& Summers, M. F. How retroviruses select their genomes. Nat. Rev. Microbiol. 3, 643-655 (2005).

52. Laham-Karam, N. \& Bacharach, E. Transduction of human immunodeficiency virus type 1 vectors lacking encapsidation and dimerization signals. J. Virol. 81, 10687-10698 (2007)

53. Shakeel, S. et al. Genomic RNA folding mediates assembly of human parechovirus. Nat. Commun. 8, 5 (2017).

54. Patel, N. et al. HBV RNA pre-genome encodes specific motifs that mediate interactions with the viral core protein that promote nucleocapsid assembly. Nat. Microbiol. 2, 17098 (2017).

55. Twarock, R. \& Stockley, P. G. RNA-mediated virus assembly: mechanisms and consequences for viral evolution and therapy. Annu. Rev. Biophys. 48, 495-514 (2019).

56. Zheng, Y. \& Kielian, M. An alphavirus temperature-sensitive capsid mutant reveals stages of nucleocapsid assembly. Virology 484, 412-420 (2015).

57. Lamb, K., Lokesh, G. L., Sherman, M. \& Watowich, S. Structure of a Venezuelan equine encephalitis virus assembly intermediate isolated from infected cells. Virology 406, 261-269 (2010).

58. Paredes, A., Alwell-Warda, K., Weaver, S. C., Chiu, W. \& Watowich, S. J. Structure of isolated nucleocapsids from venezuelan equine encephalitis virus and implications for assembly and disassembly of enveloped virus. J. Virol. 77, 659-664 (2003)

59. Yap, M. L. et al. Structural studies of Chikungunya virus maturation. Proc. Natl Acad. Sci. USA 114, 13703-13707 (2017).

60. Ahn, A., Klimjack, M. R., Chatterjee, P. K. \& Kielian, M. An epitope of the Semliki Forest virus fusion protein exposed during virus-membrane fusion. J. Virol. 73, 10029-10039 (1999).

61. Greiser-Wilke, I., Moennig, V., Kaaden, O.-R. \& Figueiredo, L. T. M. Most alphaviruses share a conserved epitopic region on their nucleocapsid protein J. Gen. Virol. 70, 743-748 (1989).

62. Kielian, M., Jungerwirth, S., Sayad, K. U. \& DeCandido, S. Biosynthesis, maturation, and acid-activation of the Semliki Forest virus fusion protein. $J$. Virol. 64, 4614-4624 (1990) 
63. Liljeström, P., Lusa, S., Huylebroeck, D. \& Garoff, H. In vitro mutagenesis of a full-length cDNA clone of Semliki Forest virus: the small 6,000-molecular-weight membrane protein modulates virus release. J. Virol. 65, 4107-4113 (1991).

64. Ashbrook, A. W. et al. Residue 82 of the Chikungunya virus E2 attachment protein modulates viral dissemination and arthritis in mice. J. Virol. 88, 12180-12192 (2014).

65. Mechold, U., Gilbert, C. \& Ogryzko, V. Codon optimization of the BirA enzyme gene leads to higher expression and an improved efficiency of biotinylation of target proteins in mammalian cells. J. Biotechnol. 116, 245-249 (2005).

66. Wan, J. J., Brown, R. S. \& Kielian, M. Berberine chloride is an alphavirus inhibitor that targets nucleocapsid assembly. mBio 11, e01382-01320 (2020).

67. Yamaji, M. et al. DND1 maintains germline stem cells via recruitment of the CCR4-NOT complex to target mRNAs. Nature 543, 568-572 (2017).

68. Benhalevy, D., McFarland, H. L., Sarshad, A. A. \& Hafner, M. PAR-CLIP and streamlined small RNA cDNA library preparation protocol for the identification of RNA binding protein target sites. Methods 118-119, 41-49 (2017).

69. Danan, C., Manickavel, S. \& Hafner, M. PAR-CLIP: a method for transcriptome-wide identification of RNA binding protein interaction sites. Methods Mol. Biol. 1358, 153-173 (2016).

70. Hafner, M. et al. PAR-CliP-a method to identify transcriptome-wide the binding sites of RNA binding proteins. J. Vis. Exp. 41, 2034 (2010).

71. Aranda, P. S., LaJoie, D. M. \& Jorcyk, C. L. Bleach gel: a simple agarose gel for analyzing RNA quality. Electrophoresis 33, 366-369 (2012).

72. Lambert, N. et al. RNA Bind-n-Seq: quantitative assessment of the sequence and structural binding specificity of RNA binding proteins. Mol. Cell 54, 887-900 (2014).

73. Martin, M. Cutadapt removes adapter sequences from high-throughput sequencing reads. EMBnet.journal 17, 10-12 (2011).

74. Hasan, S. S. et al. Cryo-EM structures of eastern equine encephalitis virus reveal mechanisms of virus disassembly and antibody neutralization. Cell Rep. 25, 3136-3147.e3135 (2018).

\section{Acknowledgements}

We thank all of the members of the Kielian lab and Dimitrios Zattas for helpful discussions and comments on the manuscript. We thank Matthew Angeliadis for technical assistance. We thank the Einstein Analytical Imaging Facility and facility members Xheni Nishku and Timothy Mendez for training and technical assistance on the electron microscope. We thank Susan Buhl and Matthew Scharff for technical assistance and use of their plate reader. This work was supported by grants to M.K. from the National Institute of General Medical Sciences (R01-GM057454) and the National Institute of Allergy and Infectious Diseases (R01-AI075647) and by Cancer Center Core Support Grant NIH/NCI P30-CA13330. R.S.B. was supported by an NIH NRSA (F32-GM122450) postdoctoral fellowship and the Charles $\mathrm{H}$. Revson Senior Fellowship in Biomedical Sciences. M.H. and D.G.A are supported by the Intramural Research Program of the
National Institute of Arthritis and Musculoskeletal and Skin Diseases. The content of this paper is solely the responsibility of the authors and does not necessarily represent the official views of the National Institute of General Medical Sciences, the National Institute of Allergy and Infectious Diseases, National Institute of Arthritis and Musculoskeletal and Skin Diseases, or the National Institutes of Health. This study was made possible (in part) by funds granted by the Charles H. Revson Foundation. The statements made and views expressed, however, are solely the responsibility of the authors.

\section{Author contributions}

R.S.B. and M.K. conceived the project. R.S.B. and D.G.A. made the PAR-CLIP libraries. D.G.A. performed the in vitro binding assay. R.S.B. performed all other experiments

M.K. and M.H. supervised the research. R.S.B. and M.K. wrote the original draft. R.S.B D.G.A., M.H., and M.K. reviewed and edited the draft.

\section{Competing interests}

The authors declare no competing interests.

\section{Additional information}

Supplementary information is available for this paper at https://doi.org/10.1038/s41467020-18447-z.

Correspondence and requests for materials should be addressed to M.K.

Peer review information Nature Communications thanks the anonymous reviewers for their contribution to the peer review of this work.

Reprints and permission information is available at http://www.nature.com/reprints

Publisher's note Springer Nature remains neutral with regard to jurisdictional claims in published maps and institutional affiliations.

Open Access This article is licensed under a Creative Commons Attribution 4.0 International License, which permits use, sharing, adaptation, distribution and reproduction in any medium or format, as long as you give appropriate credit to the original author(s) and the source, provide a link to the Creative Commons license, and indicate if changes were made. The images or other third party material in this article are included in the article's Creative Commons license, unless indicated otherwise in a credit line to the material. If material is not included in the article's Creative Commons license and your intended use is not permitted by statutory regulation or exceeds the permitted use, you will need to obtain permission directly from the copyright holder. To view a copy of this license, visit http://creativecommons.org/ licenses/by/4.0/

(C) The Author(s) 2020 